Chapter 4

\title{
Multimodal Analgesia for the Management of Postoperative Pain
}

\author{
Borja Mugabure Bujedo, Silvia González Santos, \\ Amaia Uría Azpiazu, Anxo Rubín Noriega, \\ David García Salazar and Manuel Azkona Andueza
}

Additional information is available at the end of the chapter

http://dx.doi.org/10.5772/57401

\section{Introduction}

The US Congress declared the 10-year period between January 1st, 2001, and December 31st, 2010, the decade for the control and treatment of pain, while the IASP (International Association for the Study of Pain) declared the period ending in October 2011, the year dedicated to acute pain. In spite of this measure, we must recognize that this effort has been insufficient, and that pain is one of the main health problems in the $21^{\text {st }}$ century [1]. There is no ideal analgesic regimen, as none encompasses the characteristics of a fast onset of action, good costeffectiveness profile, absence of short and long-term adverse effects, nil interaction with other drugs and/or metabolites, and ease of administration, both for the patients and healthcare personnel. Furthermore, technical deficiencies in the drug-delivery systems have contributed to a worsening of this situation, which is why, over the past few years, new and more precise mechanisms have appeared to allow us to improve the overall quality of analgesic regimens, "making old drugs new", especially those in the opioids family [2].

In spite of advances in the knowledge of the neurobiology of nociception and the physiology of systemic and spinal analgesic drugs, postoperative pain remains undertreated. Hospitalized postoperative patients should have the best access to analgesia, nevertheless, more than $1 / 3$ of these patients experience moderate to severe pain in the first $24 \mathrm{~h}$ after their procedure [2]. Further, around $60 \%$ of current surgery can be ambulatory, but in reality, almost $80 \%$ of patients complain about moderate postoperative pain. Inadequate treatment leads to an extension of the recovery time, an increase in the length of the hospitalization stay, of healthcare costs, and greater patient dissatisfaction [3]. 
The gap between the knowledge of the mechanism of pain production and the application of an effective treatment is great, and ever growing. Neither acute, nor chronic pain usually receives adequate treatment due to several reasons relating to culture, attitude, education, politics and logistics. The correct treatment of pain is considered a fundamental right of the patient; in fact, lawsuits have been launched due to the under-treatment of pain, as well as an indicator of good clinical practice and quality of care [4]. The ideal analgesic regimen must assess the risks against the benefits and consider the patient's preference, as well as the clinician's prior experience, and will be framed within a multimodal approach in order to facilitate postsurgical recovery. Effectiveness in the management of postoperative pain entails a multimodal approach involving several drugs with different mechanisms of action so as to achieve a synergistic effect and thus minimize the adverse effects of the different routes of administration [5].

The main objective of this review is to explain the multimodal approach to postoperative pain, defining the benefits and risks of the combination of the most common used analgesic drugs and techniques as well as the latest improvements in this field and experts' recommendations. For this purpose, a review on Ovid-Medline was carried out until December 2012, with the keywords: "postoperative pain", "postoperative convalescence", "multimodal analgesia", "non-steroidal anti-inflammatory drugs", "regional analgesia" and "opioids", focusing on systematic reviews with or without meta-analysis, randomized controlled trials and expert opinion articles concerning several controversial points.

\section{Pathophysiology of postoperative pain}

The study of the neurophysiology of pain [6] has produced important advances in the knowledge of the mechanism of the production of painful stimuli in the perioperative period, describing a dynamic system where multiple nociceptive afferent pathways, together with other downstream modulation mechanisms, are of relevance. Surgical incision triggers deep responses of an inflammatory nature and from the sympathetic system, which determines a first stage of peripheral sensitization that, if it is maintained over time, amplifies the transmission of the stimulus until it conditions a second stage of central sensitization. As a consequence, it leads to an increased release of catecholamines and increased oxygen consumption, with increased neuroendocrine activity, translating into hyperactivity in many organs and systems. This translates into cardiovascular, pulmonary, endocrine-metabolic, gastrointestinal, immunological and psychological complications.

There is a direct association between processes with a severe degree of postsurgical pain and the proportion of the appearance of chronic pain, such as with limb amputation (30-83\%), thoracotomy (36-56\%), gall bladder or breast surgery $(11-57 \%)$, inguinal hernia $(37 \%)$ and sternotomy (27\%) or abdominal hysterectomy (3-25\%) [7]. Chronic pain can be severe in about $2-10 \%$ of these patients representing a major largely unrecognized clinical problem. Iatrogenic neuropathic pain is probably the most important cause of long-term postsurgical pain and consequently surgical techniques that avoid nerve damage should be applied whenever 
possible. Also, early and aggressive pain therapy during the postoperative setting should be administered since the intensity of acute pain correlates with the risk of developing a persistent pain state. Finally, the role of genetic factors should be studied, since only a certain proportion of patients with intraoperative nerve damage develop chronic pain [8]. Many clinical trials have demonstrated the effectiveness of gabapentin and pregabalin administration in the perioperative period as an adjunct to reduce acute postoperative pain. However, very few clinical trials have examined their use in the prevention of chronic postsurgical pain (CPSP). Eight studies were included in a recent meta-analysis, the six of the gabapentin trials demonstrated a moderate-to-large reduction in the development of CPSP (pooled odds ratio [OR] $0.52 ; 95 \%$ confidence interval [CI], 0.27 to $0.98 ; \mathrm{P}=0.04$ ), and the two pregabalin trials found a very large reduction in the development of CPSP (pooled OR 0.09; 95\% CI, 0.02 to $0.79 ; \mathrm{P}=0.007$ ). This review supports the view that the perioperative administration of gabapentin and pregabalin is effective in reducing the incidence of CPSP but better-designed clinical trials are needed to confirm these early findings [9].

We must hence carry out a thorough treatment of dynamic postoperative pain, as it is not enough to only treat pain at rest, and to avoid other predicting factors, such as pain more than one month prior to the intervention, aggressive or repeated surgery, associated nerve injury or prior psychopathological factors [10]. Moreover, factors predisposing patients to a greater postoperative pain are young age and the type of surgery, such as orthopaedic surgery (due to the involvement of periosteum, which has a very low pain sensitivity threshold) and thoraco-abdominal surgery (due to the large involvement of the functions of the corresponding organs) [10]. The concept of pre-emptive analgesia is based on the administration, prior to surgical incision, of an analgesic in order to mitigate or prevent central hypersensitivity phenomena, aiming to reduce analgesic consumption in the postoperative period and chronic pain. However, there is great controversy regarding its efficacy. In a meta-analysis [11], sixtysix studies with data from 3, 261 patients were analysed. Fixed-effect model combined data were used and the effect size index (ES) was used as the standardized mean difference. When the data from all three-outcome measures were combined, the ES was the most pronounced for the pre-emptive administration of epidural analgesia (ES, $0.38 ; 95 \%$ confidence interval [CI], 0.28-0.47), local anaesthetic wound infiltration (ES, 0.29; 95\% CI, 0.17-0.40), and nonsteroidal anti-inflammatory-drugs (NSAIDs) administration (ES, 0.39; 95\% CI, 0.27-0.48). Whereas pre-emptive epidural analgesia resulted in consistent improvements in all threeoutcome variables, pre-emptive local anaesthetic wound infiltration and NSAIDs administration improved analgesic consumption and time to first rescue analgesic request, but not postoperative pain scores. The least proof of efficacy was found for systemic NMDA antagonist (ES, $0.09 ; 95 \%$ CI, -0.03 to 0.22 ) and opioid (ES, $-0.10 ; 95 \%$ CI, -0.26 to 0.07 ) administration, and the results remain equivocal. Epidural analgesia begun prior to the surgical stimulus and maintained for several days (2-4) in the postoperative period has previously shown to be effective in this setting, either for amputations or thoracotomy and laparotomy, focusing on the timing of the perioperative analgesia [12].

Hyperalgesia can occur after surgery either due to nervous system sensitization caused by surgical nociception (nociception-induced hyperalgesia) or as an effect of anaesthetic drugs, 
particularly opioids (opioid-induced hyperalgesia - OIH). Both are potentially undesirable and can share similar underlying mechanisms such as the involvement of excitatory amino acids via the N-methyl-D-aspartate (NMDA) receptors [13]. Hyperalgesia is characterized by a deviation down and to the left of the curve that associates the intensity of the stimulus to the degree of pain observed, so that a usually painful stimulus is perceived as a pain of greater intensity, and likewise, another stimulus that is not painful is perceived as painful (allodynia). This effect may be seen both in the peripheral and central nervous systems. Primary hyperalgesia is a consequence of the sensitization of peripheral nociceptors during the inflammatory phase which is sustained by the local ischemia and acidosis caused by thermal or mechanical stimuli in areas close to the surgical incision. Secondary hyperalgesia is, in turn, due to central sensitization by a painful afferent stimulus sustained over time that triggers a spontaneous increase in the neuronal activity of the posterior horn of the spinal cord, only manifesting when faced with mechanical stimuli in tissues far from the lesion [14].

The clinical importance of hyperalgesia lies, on the one hand, in the increased intensity of the pain, in the consumption of analgesics, in the morbidity and in the discomfort in the postoperative period, and also, in the greater presence of chronic pain, and a greater probability of developing a complex regional pain syndrome that has even been suggested [15]. Furthermore, the greatest inconvenience lies in how hard it is to quantify; this should be done against electrical stimuli on the region of the skin, as it is not usually reflected in traditional subjective pain assessment scales (visual or numeric analogic scales), and objective neuroplasticity assessment tests (Von Frey filaments) that provide complementary information for a correct adjustment of the treatment. This should be based on neuromodulator drugs like gabapentinoids (gabapentin or pregabalin), ketamine, or NSAIDs. Finally, effective perioperative blocking of nociceptive inputs from the wound with regional analgesia as well as the use of antihyperalgesic and analgesic drugs in a multimodal combination, seem to be the best way to prevent central sensitization $[14,15]$.

\section{Systemic analgesia}

\subsection{Non-steroidal-anti-inflammatory-drugs: NSAIDS}

The acceptance of the concept of multimodal analgesia and the appearance of parenteral preparations has increased the popularity of NSAIDs in the management of postoperative pain [16]. The potential beneficial effects are summarized in Table I.

The mechanism of action involves the peripheral and central inhibition of cyclooxygenase (COX) and to the reduced production of prostaglandins from arachidonic acid. Two isoenzymes have been described [17], COX-1: Constitutive, responsible for platelet aggregation, haemostasis and the protection of the gastric mucosa, but it also increases by 2-4 times in the initial inflammatory process and in the synovial fluid of chronic processes such as rheumatoid arthritis and COX-2: Induced, causing pain (by increasing by 20-80 times in the inflammation), fever and carcinogenesis (by facilitating tumour invasion, angiogenesis and metastasis). However, both forms are constitutive in the dorsal root ganglion and in the grey matter of the 


\section{IMPROVEMENT OF ANALGESIA:}

- Reduced activation and sensitization of peripheral nociceptors

- Attenuation of the inflammatory response.

- Coverage of some types of pain better than opioids (osseous pain, pain during movement and when coughing).

- Effectiveness in its use as part of a multimodal analgesia.

- Synergistic effect with opioids (reduction of opioid dose by $20 \%$ to $50 \%$ ).

- Preventive analgesia (due to a reduction of neuronal desensitization and of production of medullary prostaglandins).

\section{LESS ADVERSE EFFECTS THAN OPIOIDS:}

- Lower individual dose variability than with opioids.

- Long duration of action half-life.

- No generation of dependence or addiction.

- No respiratory depression

- Lower incidence of paralytic ileus, nausea and vomiting than with opioids.

- No production of central alterations (either cognitive or pupillary).

- $C O X-2$ : Lower incidence of GI adverse effects and a no anti-platelet activity.

Table 1. Beneficial actions attributed to NSAIDs in the appropriate management of postoperative pain $[16,17]$

spinal cord. Therefore, although the spinal administration of COX-1 inhibitors has not shown to be effective, COX-2 inhibitors (Coxib) may play an important role in central sensitization and in the anti-hyperalgesic effect by blocking the constitutive form at the medullary level and by reducing the central production of prostaglandin E-2. Although Coxib drugs present with a lower risk of gastrointestinal haemorrhage and a nil effect on platelet function, they have not been demonstrated to reduce renal complications (hypertension, oedema, nephrotoxicity) and the effects on osteogenesis, compared to non-selective NSAIDs are still controversial [16, 17, 18]. It has been proposed that COX-2 is a cardioprotective enzyme and that the cardiovascular risk associated with its inhibition is due to an alteration in the balance between prostacyclin I-2 (endothelial) and thromboxane A-2 (platelet) in favour of the latter which leads to platelet aggregation, vasoconstriction and vascular proliferation. Coxib drugs improve the side effect profile and maintain a similar analgesic power; however, the duration of the treatment with these drugs in at-risk patients, their adverse effects, cost/effectiveness and efficacy compared to that of conventional NSAIDs associated with gastric protectors and their reliability in patients who usually take anti-aggregate drugs have not yet been defined [17, 18]. On the basis of many human studies, one may conclude that perioperative COX-2 inhibitors, in standard doses, decrease opioid consumption, but it is not clear whether they decrease adverse events related to the opioids. Future investigations with different multimodal techniques may help elucidate and clarify the true benefits of perioperative COX-2 inhibitors in acute pain management strategies [18].

Celecoxib is a sulphonamide with a large volume of distribution (400 litres/200 mg), large tissue penetration, degradation through the cytochrome P450 2C9/3A4 system, and a half-life of 11 $\mathrm{h}$, with inactive metabolites. Rofecoxib is a sulphone with a volume of distribution of 86-litres/ 
$25 \mathrm{mg}$, it is metabolized by cytosolic reduction, without interacting with the cytochrome system, and its half-life is of $17 \mathrm{~h}$, with active metabolites. The equipotent dose for the treatment of acute pain is $400 \mathrm{mg}$ of celecoxib/50 $\mathrm{mg}$ of rofecoxib. This would explain the differences between COX-2/COX-1 selectivity, and the differences found in the incidence of cardiovascular adverse effects, which are greater for rofecoxib [19, 20]. The decision to withdraw this drug from the US market in September 2004 was based on a three year controlled clinical trial on the prevention of adenomatous polyposis, in which an increased relative risk of cardiovascular effects such as ischemia or myocardial infarction was found in patients who were on treatment for more than 18 months. The risk of myocardial infarction varies with individual NSAIDs. An increased risk was observed for diclofenac and rofecoxib, the latter having a clear doseresponse trend. There was a suggestion of a small increased risk with ibuprofen. Data also suggest a small-reduced risk for naproxen present only in non-users of aspirin, mainly people free of clinically apparent vascular disease [20].

Etoricoxib is a selective cyclo-oxygenase-2 (COX-2) inhibitor licensed for the relief of chronic pain in osteoarthritis and rheumatoid arthritis, and acute pain in some jurisdictions. This class of drugs is believed to be associated with fewer upper gastrointestinal adverse effects than conventional non-steroidal anti-inflammatory drugs (NSAIDs). Single dose oral etoricoxib produces high levels of good quality pain relief after surgery and the incidence of adverse events did not differ from the placebo. The $120 \mathrm{mg}$ dose is as effective as, or better than, other commonly used analgesics [21].

Parecoxib is a pro-drug used in Europe for parenteral administration in the treatment of moderate-to-severe postoperative pain. The IV administration of $40 \mathrm{mg}$ produces analgesia at $14 \mathrm{~min}$. and as it is rapidly hydrolysed in the liver into valdecoxib, it is not detected in urine. Its analgesic peak is detected after $2 \mathrm{~h}$ and its duration varies from between 5-22 h. Its usefulness in reducing pain after dental, gynaecological, abdominal, orthopaedic and cardiac surgery has been proven. The analgesic efficacy of $40 \mathrm{mg}$ IV is similar to that of ketorolac $30 \mathrm{mg}$ IV. The maximum daily dose recommended is of $80 \mathrm{mg}$ [22]. Parecoxib is contraindicated in patients with ischaemic heart disease or established cerebrovascular disease, in patients with congestive heart failure (NYHA classes II-IV), as well as in the treatment of postoperative pain after coronary by-pass surgery.

The efficacy of paracetamol or acetaminophen [23] has been proven in the treatment of moderate postoperative pain and in many other types of acute pain. It appears it could act by blocking the COX-3 detected in the cerebral cortex, thus reducing pain and fever. This third isoenzyme, which is similar to the mRNA of COX-1, has a retained intron- 1 that alters its genetic expression in humans, and it may lead to questions as to whether this is the pathway for its therapeutic action, which, centrally, could be favoured for its lower presence of endoperoxides in nerve cells. The main analgesic mechanism appears to be due to a modulation of the serotonergic system, and it is possible that it increases noradrenalin concentrations in the CNS and peripheral $\beta$-endorphins. Thus, even if the mechanism of action is not clearly understood, there is now evidence that paracetamol acts within the CNS, by inhibiting the prostaglandin synthesis, whereas it has very weak antiplatelet and anti-inflammatory effects at recommended dosages. It manifests with a potentiating effect on NSAIDs and opioids and at therapeutic 
doses it does not present with relevant adverse effects. It presents with a very favourable efficacy/tolerability ratio, which is why it has been turned into the first-line of treatment in postoperative multimodal analgesia regimens. Its peak effect in the CSF is achieved at $1-2 \mathrm{~h}$ and its concentration in this compartment remains above that of plasma after repeated doses. It has been suggested that better analgesia could be obtained with a $2 \mathrm{~g}$ starting dose instead of with the recommended dose of $1 \mathrm{~g}$. Its maximum daily dose is $4 \mathrm{~g}$, but $3 \mathrm{~g}$ per day should not be exceeded in alcohol abusers or patients with a coexisting disease causing glutathione depletion. The usual scheme of administration (1 g every 6 hours) has a less than $10 \mathrm{mg}$ sparing effect on 24 hour morphine consumption and consequently does not significantly reduce morphine side effects [24]. In a meta-analysis, seven prospective randomized controlled trials, involving 265 patients in the group with PCA (patient-controlled-analgesia) morphine plus acetaminophen and 226 patients in the group with PCA morphine alone, were selected. Acetaminophen administration was not associated with a decrease in the incidence of morphine-related adverse effects or an increase in patient satisfaction. Adding acetaminophen to PCA was associated with a morphine-sparing effect of $20 \%$ (mean, $-9 \mathrm{mg}$; CI -15 to $-3 \mathrm{mg}$; $\mathrm{P}=0.003$ ) over the first postoperative $24 \mathrm{~h}$ [24]. In a recent systematic review, it has been verified how the association of paracetamol with other NSAIDs (diclofenac, ibuprofen, ketoprofen, ketorolac, tenoxicam, rofecoxib and aspirin) improved the efficacy of paracetamol administered alone (85\% of the studies), as well as that of anti-inflammatories (64\% of the studies) [25]. The antinociception induced by the intraperitoneal co-administration of combinations of paracetamol with the NSAIDs; diclofenac, ibuprofen, ketoprofen, meloxicam, metamizole, naproxen, nimesulide, parecoxib and piroxicam was studied by isobolographic analysis in the acetic acid abdominal constriction test in mice (writhing test). As shown by isobolographic analysis, all the combinations were synergistic, the experimental ED50s being significantly smaller than the theoretically calculated ED50s. The results of this study demonstrate potent interactions between paracetamol and NSAIDs and validate the clinical use of combinations of these drugs in the treatment of pain conditions [26].

Metamizole or dipyrone is another powerful analgesic and antipyretic agent, with limited antiinflammatory power, that is broadly used in Spain, Russia, South America and Africa, but that is not marketed in the US or the United Kingdom due to the possible risk of agranulocytosis and aplastic anaemia. Other inconveniences of metamizole include the possibility of episodes of severe allergic reactions and of hypotension after its administration via IV [16]. It presents with a spasmolytic action and an efficacy that is superior to that of salicylates, which is why it is indicated in moderate to severe postoperative pain and in colic-type pain. In a systematic review [27], over $70 \%$ of participants experienced at least $50 \%$ pain relief over 4 to 6 hours with $500 \mathrm{mg}$ of oral dipyrone compared to $30 \%$ with a placebo in five studies (288 participants). Fewer participants needed rescue medication with dipyrone $(7 \%)$ than with the placebo $(34 \%$; four studies, 248 participants). There was no difference in participants experiencing at least $50 \%$ pain relief with $2.5 \mathrm{~g}$ intravenous dipyrone and $100 \mathrm{mg}$ intravenous tramadol $(70 \%$ versus 65\%; two studies, 200 participants). No serious adverse events were reported.

Diclofenac is an anti-inflammatory with a great analgesic capacity, especially after orthopaedic and traumatological surgery, due to its great penetration into inflamed tissues and synovial fluid. It is also of use in pains of a colic nature, such as renal pain. The maximum daily dose is 
of $150 \mathrm{mg}$, distributed in 2 doses, and it is important to remember that some countries only approve it for deep intramuscular use [28]. Its greatest contraindication is kidney failure and gastrointestinal bleeding disorders. A new formulation of the non-selective NSAID diclofenac sodium suitable for intravenous bolus injection has been developed using hydroxypropyl betacyclodextrin as a solubility enhancer (HPbetaCD diclofenac). HPbetaCD diclofenac intravenous bolus injection was shown to be bioequivalent to the existing parenteral formulation of diclofenac containing propylene glycol and benzyl alcohol as solubilizers (PG-BA diclofenac), which is relatively insoluble and requires slow intravenous infusion over 30 minutes. For patients with acute moderate and severe pain after abdominal or pelvic surgery, repeated 18.75 $\mathrm{mg}$ and $37.5 \mathrm{mg}$ doses of HPßCD diclofenac provided significant analgesic efficacy, as compared to a placebo. Significant analgesic efficacy was also provided by the active comparator ketorolac. Both $\mathrm{HP} \beta \mathrm{CD}$ diclofenac and ketorolac significantly reduced the need for opioids [29].

Dexketoprofen trometamol is one of the most potent "in vitro" inhibitors of prostaglandin synthesis; it is a soluble salt of the (S)-(+) right-handed enantiomer of ketoprofen. It is administered at doses of $12.5-25 \mathrm{mg}$ orally, with a fast absorption with an empty stomach, and recently has been administered at $50 \mathrm{mg}$ IV with a maximum daily dose of $150 \mathrm{mg}$ for only 48 $\mathrm{h}$, binding strongly to albumin, and with a renal excretion of inactive metabolites after glucuronidation. Ketoprofen at doses of $25 \mathrm{mg}$ to $100 \mathrm{mg}$ is an effective analgesic in moderate to severe acute postoperative pain with an NNT for at least $50 \%$ pain relief of 3.3 with a $50 \mathrm{mg}$ dose. This is similar to that of commonly used NSAIDs such as ibuprofen (NNT 2.5 for a 400 $\mathrm{mg}$ dose) and diclofenac (NNT 2.7 at a $50 \mathrm{mg}$ dose). The duration of action is about five hours. Dexketoprofen is also effective with NNTs of 3.2 to 3.6 in the dose range $10 \mathrm{mg}$ to $25 \mathrm{mg}$. Both drugs were well tolerated in single doses and its main indication is acute postoperative pain and nephritic colic [30].

Ketorolac is an anti-inflammatory with a great analgesic power, equitable to that of meperidine and even morphine, but with a roof therapeutic effect. It is absorbed orally, by IM, IV and topically through the eye, as it is well tolerated by all human tissues. It binds to plasma proteins to a degree of $99 \%$, and it's eliminated by the renal pathway as an active drug and metabolites. It is very useful in postoperative pain, of the renal colic and spastic bladder-type. It has also been used successfully in IV regional anaesthesia together with lidocaine [31]. The recommended doses are $10 \mathrm{mg}$ orally or $30 \mathrm{mg}$ parentally, with a maximum duration of five and two days, respectively. Its main adverse effects are dyspepsia and nausea, although it must be used cautiously in patients with a history of gastrointestinal bleeding. A European multicentre study that compared ketorolac with ketoprofen and naproxen used postoperatively ( $\leq 5$ days) evaluated the risk of death $(0.17 \%)$, surgical bleeding $(1.04 \%)$, gastrointestinal bleeding $(0.04 \%)$, acute kidney failure $(0.09 \%)$ and allergic reactions $(0.12 \%)$ on 11,245 patients, and found no significant differences among them [32].

It is a proven fact that NSAIDs are effective in the postoperative treatment of moderate to severe pain, but it is yet to be verified what systematic reviews suggest: that they can be as effective as opioids [5, 16, 33]. (See Table II, Oxford Listing about the efficacy of single-dose analgesics based on Systematic Reviews. NOTE: The lower the NNT, the greater the potency) 


\begin{tabular}{|c|c|c|}
\hline NSAIDS & NSAIDS + OPIOIDS & OPIOIDS \\
\hline Etoricoxib PO & Paracetamol $1 \mathrm{~g}+$ Codeine $60 \mathrm{mg}$ PO & Oxycodone PO $15 \mathrm{mg}$ \\
\hline 60 mg NNT 2.2 (1.7-3.2) & NNT $2.2(1.7-2.9)$ & NNT $2.4(1.5-4.9)$ \\
\hline 80 mg NNT 1.6 (1.5-1.8) & Paracetamol $500 \mathrm{mg}+$ Oxycodone IR & \\
\hline 180-240 mg NNT 1.5 (1.3-1.7) & 5 mg NNT $2.2(1.7-3.2)$ & \\
\hline Valdecoxib PO & Paracetamol $500 \mathrm{mg}+$ Oxycodone IR & \\
\hline 40 mg NNT 1.6 (1.4-1.8) & 10 mg NNT $2.6(2.0-3.5)$ & \\
\hline 20 mg NNT 1.7 (1.4-2.0) & Paracetamol 650 mg + Tramadol 75 & \\
\hline Parecoxib IV & mg PO NNT 2.6 (2.0-3.0) & \\
\hline 40 mg NNT 1.7 (1.3-2.4) & Paracetamol $1000 \mathrm{mg}+$ Oxycodone IR & \\
\hline 20 mg NNT 2.5 (2.0-4.8) & 10 mg PO NNT 2.7 (1.7-5.6) & \\
\hline Celecoxib PO & Paracetamol 650 mg + Tramado/ 112 & \\
\hline 200 mg NNT 3.5 (2.9-4.4) & mg PO NNT 2.8 (2.1-4.4) & \\
\hline \multicolumn{3}{|l|}{400 mg NNT $2.1(1.8-2.1)$} \\
\hline \multicolumn{3}{|l|}{ Rofecoxib PO } \\
\hline \multicolumn{3}{|l|}{50 mg NNT 2.2 (1.9-2.4) } \\
\hline Diclofenac PO, IM & Paracetamol $1000 \mathrm{mg}+$ Oxycodone IR & Morphine IM $10 \mathrm{mg}$ \\
\hline 100 mg NNT $1.8(1.5-2.1)$ & 5 mg PO NNT 3.8 (2.1-20.0) & NNT 2.9 (2.6-3.6) \\
\hline \multicolumn{3}{|l|}{50 mg NNT 2.3 (2.0-2.7) } \\
\hline \multicolumn{3}{|l|}{25 mg NNT 2.8 (2.1-4.3) } \\
\hline Ketoprofen PO & & Meperidine IM $100 \mathrm{mg}$ \\
\hline 50 mg $3.3(1.6-4.5)$ & & NNT 2.9 (2.3-3.9) \\
\hline \multicolumn{3}{|l|}{ Dexketoprofen } \\
\hline \multicolumn{3}{|l|}{10 mg PO NNT 3.2 (2.8-3.4) } \\
\hline \multicolumn{3}{|l|}{25 mg PO NNT 3.6 (2.6-4.2) } \\
\hline \multicolumn{3}{|l|}{50 mg IV similar to diclofenac IM } \\
\hline Ibuprofen PO & Paracetamol 600/650 mg + Codeine & Tapentadol PO: \\
\hline 400 mg + Paracetamol $1 \mathrm{~g}$ NNT 1.5 & 60 mgPO NNT 4.2 (3.4-5.3) & - Bunionectomy pain $(50,75,100 \mathrm{mg})$ \\
\hline$(1.4-1.7)$ & Paracetamol 650 mg + & NNT $3.6-3.8-2.5$ \\
\hline 200 mg + Paracetamol 500 mg NNT & Dextropropoxifen 65 mg PO & - Dental pain $(50,75,100,200$ mg) \\
\hline $1.6(1,5-1.8)$ & NNT $4.4(3.5-5.6)$ & NNT $13,5,2,3$ \\
\hline \multicolumn{3}{|l|}{600 mg NNT 2.4 (1.9-3.3) } \\
\hline \multicolumn{3}{|l|}{400 mg NNT 2.7 (2.5-3.0) } \\
\hline \multicolumn{3}{|l|}{200 mg NNT 3.3 (2.8-4.0) } \\
\hline \multicolumn{3}{|l|}{ Flurbiprofen PO } \\
\hline \multicolumn{3}{|l|}{100 mg NNT 2.5 (2.0-3.1) } \\
\hline \multicolumn{3}{|l|}{50 mg NNT 2.7 (2.3-3.3) } \\
\hline \multicolumn{3}{|l|}{ Metamizole PO, IV } \\
\hline \multicolumn{3}{|l|}{500 mg NNT 2.4 (1.9-3.2) } \\
\hline $2 \mathrm{~g}$ IV similar to $100 \mathrm{mg}$ tramadol & & \\
\hline
\end{tabular}




\begin{tabular}{|c|c|c|}
\hline NSAIDS & NSAIDS + OPIOIDS & OPIOIDS \\
\hline Ketorolac PO $10 \mathrm{mg}$ & Aspirin $650 \mathrm{mg}+$ Codeine $60 \mathrm{mg} P O$ & Tramadol PO $100 \mathrm{mg}$ \\
\hline NNT $2.6(2.3-3.1)$ & NNT $5.3(4.1-7.4)$ & NNT 4.8 (3.4-8.2) \\
\hline Ketorolac IM 30 mg & & Tramadol PO $50 \mathrm{mg}$ \\
\hline NNT 3.4 (2.5-4.9) & & NNT 7.1 (4.6-18) \\
\hline Naproxen Na PO 550 mg & Paracetamol 325 mg + Oxycodone IR5 & Dextropropoxifen PO $65 \mathrm{mg}$ \\
\hline NNT 2.6 (2.2-3.2) & mg PO NNT 5.5 (3.4-14.0) & NNT 7.7 (4.6-22) \\
\hline \multicolumn{3}{|l|}{ Piroxicam 20 mg PO } \\
\hline \multicolumn{3}{|l|}{ NNT $2.7(2.1-3.8)$} \\
\hline Paracetamol PO & Paracetamol 300 mg + Codeine 30 & Dihydrocodeine PO $30 \mathrm{mg}$ \\
\hline 1 g NNT 3.8 (3.4-4.4) & mg PO NNT 5.7 (4.0-9.8) & NNT $8.1(4.1-540)$ \\
\hline 650 mg NNT $5.3(4.1-7.2)$ & & Codeine PO $60 \mathrm{mg}$ \\
\hline Aspirin PO & & NNT 9.1 (6.0-23.4) \\
\hline \multicolumn{3}{|l|}{1200 mg NNT 2.4 (1.9-3.2) } \\
\hline \multicolumn{3}{|l|}{$1 \mathrm{~g} \mathrm{NNT} 4.0$ (3.2-5.4) } \\
\hline \multicolumn{3}{|l|}{650 mg NNT 4.4 (4.0-4.9) } \\
\hline \multicolumn{3}{|l|}{ PO: Per Os (orally) } \\
\hline \multicolumn{3}{|l|}{ IM: Intramuscularly } \\
\hline \multicolumn{3}{|l|}{ V: Intravenously } \\
\hline \multicolumn{3}{|l|}{ IR: Immediate release } \\
\hline (Between brackets after NN & fidence interval) & \\
\hline
\end{tabular}

Table 2. Relative efficacy of several analgesics according to the $n n t$ in acute pain $[5,16,33]$ (NNT: Number of patients necessary to treat in order to achieve a $50 \%$ relief of moderate to severe postoperative pain after a single dose)

\subsection{Opioids}

Opioids are the drugs with the greatest known analgesic efficacy. This is because their action is the result of a combined interaction on four types of receptors in turn divided into several subtypes $\left(\mu_{1-3}, \delta_{1-2}, \kappa_{1-3}\right.$, ORL-1) that are located at different levels of the nerve axis, from the cerebral cortex to the spinal cord, and in some peripheral locations, and that intervene both in afferent and efferent mechanisms of nociceptive sensitivity. They are also a part of the endogenous neuromodulator system of pain, and are associated with the adrenergic, serotonergic and GABAergic system [16].

Opioids produce a high degree of analgesia, without a roof effect, but are limited by the appearance of side effects such as respiratory depression, nausea and itching. Their parenteral use in moderate to severe pain achieves a good analgesic effect in a short period of time; the intravenous route being preferable to the intramuscular route due to their greater bioavailability. The oral route with sustained-release drugs is also showing its usefulness in this setting $[34,35]$. The features of the main parenteral opioids are summarized in table III. 


\begin{tabular}{|c|c|c|c|c|c|c|c|}
\hline OPIOIDS & $\begin{array}{l}\text { Onset of } \\
\text { action } \\
\text { (min) }\end{array}$ & $\begin{array}{l}\text { Peak effect } \\
\text { (min) }\end{array}$ & $\begin{array}{l}\text { Duration } \\
\text { of the } \\
\text { clinical } \\
\text { effect }(h)\end{array}$ & $\begin{array}{c}\text { Potency } \\
\text { compared to } \\
\text { morphine }\end{array}$ & $\begin{array}{l}\text { IV-PCA bolus } \\
\text { dose }\end{array}$ & $\begin{array}{l}\text { Time of } \\
\text { closure of } \\
\text { IV-PCA } \\
\text { (min) }\end{array}$ & $\begin{array}{c}\text { Continuous IV } \\
\text { infusion * }\end{array}$ \\
\hline Morphine ${ }^{* \star}$ & $2-4$ & $15-20$ & 2 & 1 & $1-2 \mathrm{mg}$ & $6-10$ & 0-2 mg h-1 \\
\hline Hydromorphone & $2-3$ & $10-15$ & 2 & 5 & $0.2-0.4 \mathrm{mg}$ & $6-10$ & $0-0.4 \mathrm{mg} \mathrm{h}-1$ \\
\hline Meperidine $* \star \star$ & 10 & 30 & $3-4$ & $1 / 10$ & $10-20 \mathrm{mg}$ & $6-10$ & $0-20 \mathrm{mg} \mathrm{h}-1$ \\
\hline Fentanyl & $1-2$ & 5 & $1-2$ & 100 & $20-50 \mu \mathrm{g}$ & $5-10$ & $0-60 \mu \mathrm{g} \mathrm{h}-1$ \\
\hline Sufentanil & 1 & 5 & 1 & 1000 & 4-6 $\mu \mathrm{g}$ & $5-10$ & 0-8 $\mu \mathrm{g} \mathrm{h-1}$ \\
\hline Tramadol & 10 & 35 & $4-6$ & $1 / 10$ & $10-20 \mathrm{mg}$ & $6-10$ & $0-20 \mathrm{mg} \mathrm{h}-1$ \\
\hline Methadone & $2-3$ & $5-6$ & $6-12$ & 1 & $0.5 \mathrm{mg}$ & $10-15$ & $0-0.5 \mathrm{mg} \mathrm{h}-1$ \\
\hline
\end{tabular}

* Not recommended for initial programming except in patients undergoing chronic treatment with opioids or insufficient analgesia with PCA alone.

\footnotetext{
**Not recommended in patients with serum creatinine levels $>2 \mathrm{mg} / \mathrm{dL}$, due to an accumulation of the active metabolite morphine-6-glucuronide.

*** Contraindicated in patients with kidney failure, convulsive disorders (due to their neurotoxic metabolite normeperidine), or patients who take MAOIs due to the risk of malignant hyperthermia syndrome. Only recommended in patients with intolerance to all other opioids.
}

Table 3. Recommended dosage for most common IV opioids [5, 16, 34, 35]

\subsection{Opioids with special characteristics}

Tramadol [36] is a synthetic opioid with a weak affinity for receptor $\mu(6,000$ times lower than morphine) and also for receptors $\kappa$ and $\sigma$; it presents with a non-opioid mechanism, as it inhibits the central reuptake of serotonin and adrenaline, and has mild properties as a local peripheral anaesthetic. It produces a smaller number of side effects, such as nausea, due to a lower potency compared to morphine (1/5-1/10 depending on whether its administration is oral or parenteral) and it has an active metabolite [M1 (mono-O-desmethyltramadol)] with a greater affinity for opioid receptors than the original compound, which is why it contributes to the overall analgesic effect. It has shown its usefulness in a large variety of processes with moderate pain, with a dose of $100 \mathrm{mg} / 8 \mathrm{~h}$ IV recommended in the postoperative period. The efficacy of tramadol for the management of moderate to severe postoperative pain has been demonstrated in both inpatients and day surgery patients. Most importantly, unlike other opioids, tramadol has no clinically relevant effects on respiratory or cardiovascular parameters. It may prove particularly useful in patients with poor cardiopulmonary function, including the elderly, the obese and smokers, in patients with impaired hepatic or renal function, and in patients in whom NSAIDs drugs are not recommended or need to be used with caution. Parenteral or oral tramadol has proved to be an effective and well-tolerated analgesic agent in the perioperative setting.

Oxycodone [37] is a semisynthetic pure agonist derived from the natural opioid alkaloid thebaine, which is becoming the most used opioid in North America for the treatment of moderate to severe pain, as its pharmacodynamics are similar to those of morphine. Because 
its chemical structure only varies in a $\mathrm{CH} 3$ group in position 3, and an oxygen in position 6, it has certain pharmacokinetic advantages over morphine. Its administration, aside from analgesia, produces anxiolysis, euphoria, a sensation of relaxation, and inhibits coughing. It is available as immediate-release and sustained-release oral tablets, releasing $38 \%$ during the first two hours and the rest during the following 6-12 h, which is why they must be swallowed without chewing, to avoid an overdose. It differs from morphine in terms of its greater oral bioavailability (60-87\% in the retarded form, and almost $100 \%$ in the immediate-release form), a slightly greater half-life (3-5 h) and in its liver metabolism, which occurs by means of the cytochrome P-450 (CPY2D6) rather than by glucuronidation, which is why it can interact with sertraline and fluoxetine, potent inhibitors of said enzyme. It reaches a plasma steady state after 24-36 h of treatment. It is metabolized mainly into noroxycodone, which has a relative analgesic potency of 0.6 and to a lesser extent, in oxymorphone which has a high analgesic power, both of which are eliminated by the kidney. The plasma clearance for adults is of 0.8 $\mathrm{L} / \mathrm{min}$, and about $40 \%$ binds to proteins. Its administration must not be adjusted with respect to age, although it is reduced by $20-50 \%$ in patients with liver or kidney failure and concomitant treatment with other CNS depressants, such as benzodiazepines. A better risk/benefit ratio in the postoperative period appears to be associated with the use of ibuprofen or paracetamol and it has a neuropathic pain efficacy due to its " $\kappa$-agonist" action. As a treatment guide, 10 $\mathrm{mg}$ of oxycodone are equal to $20 \mathrm{mg}$ of oral morphine. Oxycodone is highly effective and well tolerated in different types of surgical procedures and patient groups, from preterm to aged patients. In the future, the use of trans mucosal administration and enteral oxycodonenaloxone controlled-release tablets is likely to increase, and an appropriate concurrent use of different enteral drug formulations will decrease the need for more complex administration techniques, such as intravenous patient-controlled analgesia [38].

Tapentadol [39] is a new mixed analgesic of dual central action, $\mu$-opioid agonist and noradrenalin reuptake inhibitor. It is 2-3 times less potent than morphine, but it is in turn, twice as potent as tramadol. It was approved in November 2008 by the FDA for the treatment of moderate to severe pain in adult patients. It is available in immediate-release (IR) tablets of 50, $75,100,150 \mathrm{mg}$, with a half-life of 4-6 h and a maximum daily dose of $600 \mathrm{mg}$. A 12-h sustainedrelease presentation has recently been marketed for the management of chronic pain. It has a better safety profile for nausea and/or vomiting and constipation compared to oxycodone IR and also has a significantly lower rate of treatment discontinuation. It has been successfully tested after otorhinolaryngological and dental surgery, in chronic osteoarticular pain, both of the rachis and is associated with knee and hip arthrosis. The observed efficacy across different pain models and favourable gastrointestinal tolerability profile associated with tapentadol IR indicate that this novel analgesic is an attractive treatment option for the relief of moderateto-severe acute pain [40].

\subsection{Non-opioid analgesic coadjutants}

Good pain control after surgery is important in preventing negative outcomes such as tachycardia, hypertension, myocardial ischemia, decrease in alveolar ventilation and poor wound healing. Exacerbations of acute pain can lead to neural sensitization and the release of 
mediators both peripherally and centrally. Clinical wind up occurs as a consequence of the processes of N-Methyl D-Aspartate (NMDA) activation, wind up central sensitization, the long-term potentiation of pain and transcription-dependent sensitization. Advances in the knowledge of molecular mechanisms have led to the development of multimodal analgesia and new pharmaceutical products to treat postoperative pain. They include extended-release epidural morphine and analgesic adjuvants such as capsaicin, ketamine, gabapentin, pregabalin, dexmedetomidine and tapentadol. Newer postoperative patient-controlled analgesia (PCA) in modes such as intranasal, regional, transdermal, and pulmonary presents another interesting avenue of development [41].

NMDA-antagonist drugs are used as modulators of pain, hyperalgesia and allodynia after surgical trauma. Ketamine is involved in opioid, cholinergic and monoaminergic systems; it may act on sodium channels, although the optimal dose and route of administration are yet to be defined. It has been tested as an analgesic potentiation drug, and in a systematic review on 2, 240 patients [42], it was verified that, in the treatment of acute postoperative pain at sub anaesthetic doses $(0.1-0.25 \mathrm{mg} / \mathrm{kg})$, either IV, IM or epidural $(0.5-1 \mathrm{mg} / \mathrm{kg})$, it is effective in reducing morphine consumption during the first $24 \mathrm{~h}$ after surgery, and reducing nausea and vomiting with a low incidence of side effects. Further, intravenous ketamine is an effective adjunct for postoperative analgesia. Particular benefit was observed in painful procedures, including upper abdominal, thoracic and major orthopaedic surgeries. The analgesic effect of ketamine was independent of the type of intraoperative opioid administered, the timing of ketamine administration, and the ketamine dose [43]. Despite using less opioid, 25 out of 32 treatment groups (78\%) experienced less pain than the placebo groups at some point postoperatively when ketamine was efficacious. This finding implies an improved quality of pain control in addition to decreased opioid consumption. Hallucinations and nightmares were more common with ketamine but sedation was not. When ketamine was efficacious for pain, postoperative nausea and vomiting were less frequent in the ketamine group. The dosedependent role of ketamine analgesia could not be determined. Dextromethorphan (40-120 mg IM) and amantadine (200 mg IV) are other drugs of this group that have been used with varying efficacy [16].

Agonists of $\alpha_{2}$-adrenergic receptors, such as clonidine (2-8 $\left.\mu \mathrm{g} / \mathrm{kg} \mathrm{IV}\right)$ and dexmedetomidine (2.5 $\mu \mathrm{g} /$ $\mathrm{kg}$ IM) enhance the analgesic and sedative effects of opioids centrally, at the level of the locus coeruleus and of the posterior medullary horn, respectively, but its side effects such as hypotension and bradycardia limit their routine use intravenously or through the medulla. A very recent systematic review and meta-analysis [44], looked at 30 relevant studies (1, 792 patients, 933 received clonidine or dexmedetomidine). There was evidence of postoperative morphine sparing at $24 \mathrm{~h}$; the weighted mean difference was $-4.1 \mathrm{mg}$ (95\% confidence interval, -6.0 to $-2.2)$ with clonidine and $-14.5 \mathrm{mg}$ (-22.1 to -6.8) with dexmedetomidine. There was also evidence of a decrease in pain intensity at $24 \mathrm{~h}$; the weighted mean difference was $-0.7 \mathrm{~cm}(-1.2$ to -0.1$)$ on a $10 \mathrm{~cm}$ visual analogic scale with clonidine and $-0.6 \mathrm{~cm}(-0.9$ to -0.2$)$ with dexmedetomidine. The incidence of early nausea was decreased with both (number needed to treat, approximately nine). Clonidine increased the risk of intraoperative (number needed to harm, approximately nine) and postoperative hypotension (number needed to harm, 20). Dexmedetomidine 
increased the risk of postoperative bradycardia (number needed to harm, three). Recovery times were not prolonged. No trial reported on chronic pain or hyperalgesia.

Gabapentin and pregabalin, structural analogues of $\gamma$-amino butyric acid, are the first-line treatment for neuropathic pain, and their usefulness in postoperative pain is due to their action on the $\alpha_{2} \delta-1$ subunit of voltage-dependent calcium channels of the posterior medullary horn. Their oral administration, and their central adverse effects, such as dizziness and somnolence, limit their use. Which is why their effective dose and treatment duration are yet to be defined. Their greatest usefulness lies in their ability to reduce the consumption of opioids in the postoperative period, as well as to reduce pain in movement and quality of sleep, which is why it is being used successfully in orthopaedic surgery, improving rehabilitation [45]. They are also useful in patients who are used to opioids by reducing their consumption in the postoperative period. They have also recently shown their usefulness in the prevention of postsurgical chronic pain [9]. In a recent metaanalysis [46], pregabalin administration reduced the amount of postoperative analgesic drugs $(30.8 \%$ of non-overlapping values - odds ratio=0.43). There was no effect with 150 , and 300 or $600 \mathrm{mg} /$ day provided identical results. Pregabalin increased the risk of dizziness or light-headedness and of visual disturbances, and decreased the occurrence of postoperative nausea and vomiting (PONV) in patients who did not receive anti-PONV prophylaxis. The authors concluded that the administration of pregabalin during a short perioperative period provides additional analgesia in the short term, but at the cost of additional adverse effects. The lowest effective dose was calculated as $225-300 \mathrm{mg} /$ day.

Postoperative nausea and vomiting are the most common complications after anaesthesia and surgery, and both female sex and laparoscopic technique are risk factors. It is certainly of a remarkably high incidence after laparoscopic gynaecological surgery, which is reported as being at nearly $70 \%$ within the first postoperative 24 hours. Corticoids have analgesic and antiinflammatory properties due to the joint inhibition of cyclooxygenase and lipoxygenase, and it has been shown that the preoperative use of dexamethasone (4-8 $\mathrm{mg}$ IV) also prevents the appearance of postoperative vomiting and nausea, especially after laparoscopy. In a recent meta-analysis [47], prophylactic dexamethasone administration decreased the incidence of nausea and vomiting after laparoscopic gynaecological operations in post-anaesthesia care units and within the first postoperative 24 hours. In a review of the current mechanisms for reducing postoperative pain, nausea and vomiting, epidural anaesthesia did not reduce the length of a hospital stay or the incidence of PONV despite reducing pain intensity and ileus. NSAIDs are more effective than paracetamol in reducing postoperative opioid consumption and PONV, while dexamethasone and 5-HT3 antagonists are both effective in reducing PONV [48]. Dehydrobenzperidol is also used as a first-line agent in the treatment of postoperative vomiting and in a quantitative systematic review of randomized controlled trials of 2, 957 patient's doses below $1 \mathrm{mg}$ was determined as the optimal IV dose. Two patients receiving $0.625 \mathrm{mg}$ of droperidol had extrapyramidal symptoms. Cardiac toxicity data were not reported. The authors concluded that because adverse drug reactions are likely to be dose-dependent, there is an argument to stop using doses of more than $1 \mathrm{mg}$ [49]. 
In a meta-analysis of 1, 754 patients, it has been verified that the perioperative infusion of lidocaine [50] reduced the intensity of pain and the consumption of opioids postoperatively, the incidence of paralytic ileus and of nausea and vomiting, as well as the length of hospital stay. The efficacy was greater in patients who underwent abdominal surgery. Considering that in some cases, toxic levels were detected, and that adverse effects were not collected systematically in all the studies, we must establish a safety range before recommending their systematic use. In another recent systematic review of 764 patients, having open and laparoscopic abdominal surgery, as well as ambulatory surgery patients [51], intravenous perioperative infusion of lidocaine resulted in significant reductions in postoperative pain intensity and opioid consumption. Pain scores were reduced at rest and with coughing or movement for up to 48 hours postoperatively. Opioid consumption was reduced by up to $85 \%$ in lidocainetreated patients when compared with controls. The infusion of lidocaine also resulted in earlier return of bowel function, allowing for earlier rehabilitation and a shorter duration of hospital stay. First flatus occurred up to 23 hours earlier, while first bowel movement occurred up to 28 hours earlier in the patients treated with lidocaine. The duration of the hospital stay was reduced by an average of 1.1 days in the patients treated with lidocaine. The administration of an intravenous lidocaine infusion did not result in toxicity or clinically significant adverse events. Lidocaine had no impact on postoperative analgesia in patients undergoing tonsillectomy, total hip arthroplasty or coronary artery bypass surgery. Systemic lidocaine also improves the postoperative quality of recovery in patients undergoing outpatient laparoscopy. In a recent study [52], patients who received lidocaine had less opioid consumption, which was translated to a better quality of recovery. The authors concluded that lidocaine is a safe, inexpensive and effective strategy for improving the quality of recovery after ambulatory surgery.

IV Magnesium has been reported to improve postoperative pain, however, the evidence is inconsistent. The objective of a very recent quantitative systematic review was to evaluate whether or not the perioperative administration of IV magnesium can reduce postoperative pain. Twenty-five trials comparing magnesium with a placebo were identified. Apart from the mode of administration (bolus or continuous infusion), perioperative magnesium reduced cumulative IV morphine consumption by $24.4 \%$ (mean difference: $7.6 \mathrm{mg}$, $95 \% \mathrm{CI}-9.5$ to $-5.8 \mathrm{mg} ; \mathrm{p}<0.00001)$ at $24 \mathrm{~h}$ postoperatively. Numeric pain scores at rest and on movement at $24 \mathrm{~h}$ postoperatively clearly improved and both were reduced by 4.2 ( $95 \% \mathrm{CI}-6.3$ to $-2.1 ; \mathrm{p}<0.0001)$ and $9.2(95 \% \mathrm{CI}-16.1$ to $-2.3 ; \mathrm{p}=0.009)$ out of 100 , respectively. The authors concluded that perioperative IV magnesium reduces opioid consumption and, to a lesser extent, pain scores, in the first 24 h postoperatively, without any reported serious adverse effects [53].

Non-pharmacological techniques, such as transcutaneous electrical nerve stimulation (TENS), which works by activating the opioid receptors and thick $A \beta$ fibres, auricular acupuncture, music therapy or psychotherapy, may also be useful in the postoperative period, but more studies are needed to verify their efficacy as coadjutant to pharmacological therapy [54]. 


\section{Patient-controlled analgesia}

\subsection{IV-PCA}

Relief of acute pain during the immediate postoperative period is an important task for anaesthesiologists. Morphine is widely used to control moderate-to-severe postoperative pain and the use of small IV boluses of morphine in the post-anaesthesia care unit (PACU) allows for a rapid titration of the dose needed for adequate pain relief. The essential principle of a titration regimen must be to adapt the morphine dose to the pain level. Although morphine would not appear to be the most appropriate choice for achieving rapid pain relief, this is the only opioid assessed in many studies of immediate postoperative pain management using titration. More than $90 \%$ of the patients achieve pain relief using a protocol of morphine titration (2-3 mg/ $5 \mathrm{~min}$.) and the mean dose required to obtain pain relief is $12 \mathrm{mg}$, after a median of four boluses. Sedation is frequent during IV morphine titration and should be considered as a morphine-related adverse event and not evidence of pain relief. The incidence of respiratory depression is very low when the criteria for limiting the dose of IV morphine are enforced. Morphine titration can be used with caution in elderly patients, in children, or in obese patients. In real practice, morphine titration allows the physician to meet the needs of individual patients rapidly and limits the risk of overdose making this method the first step in postoperative pain management [55].

The introduction of patient-controlled analgesia (PCA) has provided us a very useful tool in the adjustment of opioid doses within a broad range of postoperative needs, in turn minimizing adverse effects. Patients can self-administer a rescue dose, with or without a background regimen, thus maintaining plasma therapeutic levels. The basis of the treatment consists of a period of closure after the administered bolus in which a new administration is not allowed, thus avoiding the appearance of side effects, such as excessive sedation or respiratory depression [35].

In a practical sense [35], it is advised to administer 2-4 mg of morphine IV every 5-10 min. in the post anaesthetic recovery unit until the pain is controlled, and then start with $1 \mathrm{mg}$ every 6-8 $\mathrm{min}$, without a baseline infusion. If the patient does not achieve an adequate analgesia, the dose of the bolus will be increased to $1.5-2 \mathrm{mg}$ and, as a last resort, a continuous infusion of 1-2 $\mathrm{mg} / \mathrm{h}$ will be implemented, as long as it does not constitute $>50 \%$ of the total administered dose (see fig. $\mathrm{n}^{\mathrm{o}} 1$ ). In case of patients with chronic opioid treatment, this opioid infusion could be of up to $80 \%$. The total dose to be scheduled may be calculated according to the rule $\mathrm{mg} /$ day/morphine $=100$ - age. The systematic review showed a better analgesic quality, together with a lesser morbidity, compared to other analgesic IV regimens without PCA, but there were no differences in the total consumption of opioids, side effects or days of hospital stay. The incidence of adverse effects, such as respiratory depression $(<0.5 \%)$ does not seem to differ from other routes of opioids administration, such as the parenteral or neuraxial routes, and it is lower in the pure form of IV PCA. 


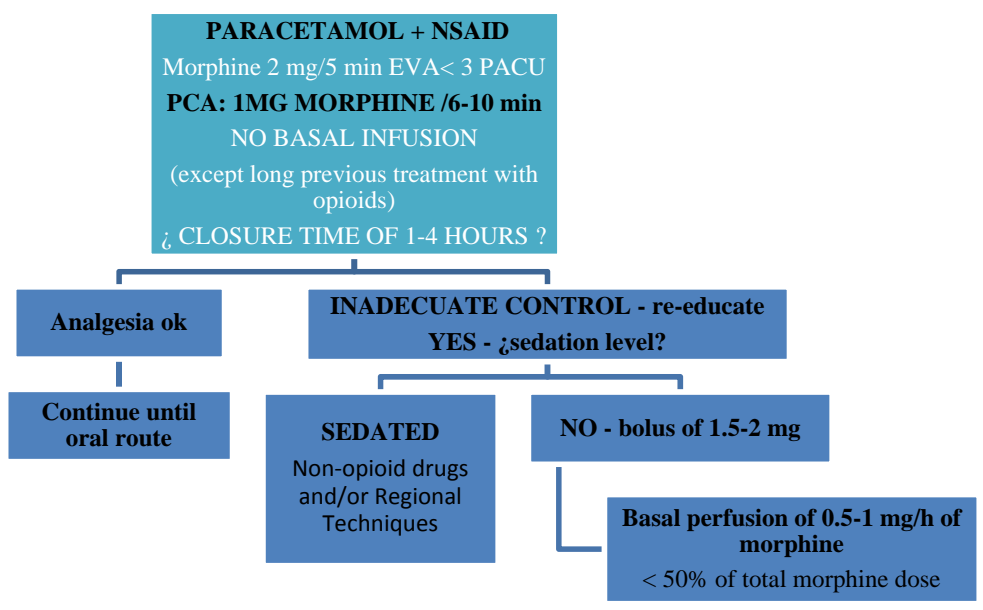

Figure 1. Titration of IV morphine in bolus or PCA in the PACU $[35,55]$

\subsection{Transdermal PCA}

Transdermal Iontophoresis [56] is a drug delivery system by which a molecule with an electrical charge penetrates through the skin in the presence of an electric field. There is a need for an active infusion system, either local or systemic, that delivers lipophilic drugs, composed of small, positively charged particles. It has been tested with transdermal fentanyl in a system similar to a credit card, with an autonomous battery, and a button for the administration of boluses, placed on the arm or on the chest. The administered dose is prefixed at $40 \mu \mathrm{g}$, with a closure of $10 \mathrm{~min}$, and with a limit of 80 doses a day and/or $24 \mathrm{~h}$ of treatment, whichever occurs first. The on-demand dosing and pharmacokinetics of this system differentiate it from the passive transdermal formulation of fentanyl designed for the management of chronic pain. Its results appear to be comparable to morphine in IV PCA in the treatment of acute postoperative pain, with a good-excellent overall satisfaction of $74-80 \%$, and with a similar incidence of adverse effects, being nausea the most frequent in almost $40 \%$ of the patients The use of this system may serve as an alternative modality for the management of acute pain without increasing such adverse effects as bleeding, intravenous catheter infiltration, or manual pump malfunction.

\subsection{Intranasal PCA}

There is also the possibility of carrying out a patient controlled intranasal analgesia (PCINA) [57] with a rapid absorption of opioids. Intranasal drug administration is an easy, well-tolerated, non-invasive trans mucosal route that avoids first-pass metabolism in the liver. The nasal mucosa provides an extensive, highly vascularized surface of pseudo stratified ciliated epithelium. It secretes mucus that is subjected to mucociliary movement that can affect the duration of the contact between the drug and the surface. Absorption is influenced by 
anatomical and physiological factors as well as by properties of the drug and the delivery system. The drug most used is fentanyl at similar doses to intravenous route, but other opioids have been used to treat acute pain like meperidine, diamorphine and butorphanol. The adverse systemic effects are similar to those described for intravenous administration, the most common being drowsiness, nausea and vomiting. Local effects reported are a burning sensation with meperidine and a bad taste.

\subsection{Patient-controlled regional analgesia}

Patient-controlled regional analgesia (PCRA) [58] encompasses a variety of techniques that provide effective postoperative pain relief without systemic exposure to opioids. Using PCRA, patients control the application of pre-programmed doses of local anaesthetics, most frequently ropivacaine or bupivacaine (occasionally in combination with an opioid), via an indwelling catheter, which can be placed in different regions of the body depending on the type of surgery. Infusions are controlled either by a staff-programmed electronic pump (similar to that used for IV PCA) or a disposable elastomeric pump. An elastomeric pump is a device that has a distensible bulb inside a protective bulb with a built-in filling port, delivery tube and bacterial filter. Analgesia can be delivered directly into a surgical incision (incisional PCRA), intraarticular (IA), tissue (IA PCRA), or perineural site (perineural PCRA).

In recent years, continuous peripheral nerve blockade has gained increasing acceptance as a safe and effective technique that provides better analgesia than opioids. A meta-analysis [59] that compared systemic opioids with regional peripheral techniques confirms a superior analgesia in the latter; regardless of whether they are used in the form of a single bolus or in a continuous infusion. In this review, perineural analgesia provided better postoperative analgesia compared with opioids $(\mathrm{P}<0.001)$. This effect was seen for all time periods measured for both mean visual analogic scale (VAS) and maximum VAS at $24 \mathrm{~h}(\mathrm{P}<0.001), 48 \mathrm{~h}(\mathrm{P}<$ 0.001 ), and $72 \mathrm{~h}$ (mean VAS only) $(\mathrm{P}<0.001)$ postoperatively. Perineural catheters provided superior analgesia to opioids for all catheter locations and time periods $(\mathrm{P}<0.05)$. Nausea/ vomiting, sedation and pruritus all occurred more commonly with opioid analgesia $(\mathrm{P}<0.001)$. A reduction in opioid use was noted with perineural analgesia $(\mathrm{P}<0.001)$. In spite of this, the overall benefit to the prognosis of postoperative patients has not been statistically proven.

\subsection{Patient-controlled epidural analgesia}

Patient-controlled epidural analgesia (PCEA) allows for an individualized postoperative regimen that reduces pharmacological requirements, improves the degree of satisfaction and provides a higher analgesic quality. In series of more than 1, 000 patients, 90\% were satisfied, with a VAS score of 1 at bed rest to 4 in motion. The presence of side effects was similar to the continuous epidural technique, standing out: itching $(16.7 \%)$, nausea $(14.8 \%)$, sedation $(13.2 \%)$, hypotension $(6.8 \%)$, motor block $(2 \%)$ and respiratory depression $(0.3 \%)$. The specific site of action of LAs is located at the level of the sheath of spinal nerve roots, the ganglion of the dorsal root and through the meninges in the spinal cord itself. The LAs most used are bupivacaine ( $\leq$ $0.125 \%)$, ropivacaine $(\leq 0.20 \%)$, and levobupivacaine $(\leq 0.125 \%)$, together with fentanyl $(2-5$ $\mu \mathrm{g} / \mathrm{mL})$ or sufentanil $(0.5-1 \mu \mathrm{g} / \mathrm{mL})$ which enhance their analgesic action and allow for a 
reducing of their total dose [60]. This route of administration has proven to be superior to the IV PCA formula with opioids. Continuous epidural techniques include the benefits of the metameric localized delivery of analgesic drugs with extended delivery in infusion and the capability to adjust the optimal degree of quality and depth in each patient, producing a sensitive postoperative block, with a minimal compromise to movement [61]. The combined use of regional-general anaesthesia improves the immediate recovery after surgery, and allows for an analgesic control of a higher quality than that offered by systemic opioids [62]. The location of the epidural catheter must be, whenever technically possible, metameric to the surgical zone, as it has been demonstrated that a thoracic catheter for thoraco-abdominal surgery reduces cardiorespiratory morbidity and mortality, improves analgesic quality and reduces the incidence of adverse effects such as urine retention and motor block [63].

A broad meta-analysis of data from 141 randomized controlled trials, which studied a total of 9, 559 patients, showed that the use of epidural or spinal anaesthesia was associated with a $30 \%$ decrease in 30 day mortality, in addition to other beneficial effects such as a $55 \%$ decrease in the incidence of pulmonary embolism, a 39\% decrease in pneumonia, a $50 \%$ decrease in transfusion requirements, and a $44 \%$ decrease in deep venous thrombosis. There was also evidence of further benefits such as a decrease in the risk of respiratory depression, myocardial infarction and renal failure [64]. However, data from more recent studies in patients undergoing major surgery failed to show any decrease in mortality with perioperative epidural analgesia when compared with a combination of general anaesthesia and the use of systemic opioids [65]. Further, an Australian multicentre study (The Master Trial), on epidural anaesthesia in abdominal surgery in high-risk patients, on 888 cases collected over six years (1995-2001) did not show such beneficial effects. There was no reduction in the morbidity in the group receiving epidural administration compared to the control group with opioids and parenteral administration, and the mortality at 30 days was similar $(4.3 \%$ in the control versus $5.1 \%$ in the group with epidural administration). Only acute respiratory failure (ARF) was less frequent in the epidural group (23\% in epidural versus $30 \%$ in the control, $p=0.02)$. An NNT of 15 patients was calculated to achieve the prevention of an ARF episode. The pain score was lower and statistically significant in the epidural group, although the VAS was only reduced by $1 \mathrm{~cm}$ in the scale $0-10 \mathrm{~cm}$ [66].

For catheter placement, the loss of resistance using saline has become the most widely used method. Patient positioning, the use of a midline or paramedian approach, and the method used for catheter fixation can all influence the success rate. When using equipotent doses, the difference in clinical effect between bupivacaine and the newer isoforms levobupivacaine and ropivacaine appears minimal. With continuous infusion, the dose is the primary determinant of epidural anaesthesia quality, with volume and concentration playing a lesser role. The addition of adjuvants, especially opioids and epinephrine, may substantially increase the success rate of epidural analgesia. The use of patient-controlled epidural analgesia (PCEA) with background infusion appears to be the best method for postoperative analgesia [67].

In spite of what was demonstrated above, the thoracic epidural with a local anaesthetic and opioid is the technique of choice for reducing the consumption of IV opioids in the postoperative period for high-risk patients, patients undergoing open vascular and major thoraco- 
abdominal surgery [68], but some authors question the routine use of this mode of analgesia in the postoperative period for patients having abdominal surgery [69] or thoracic surgery in favour of a paravertebral blockade (PVB)[70]. There is also some evidence that the use of epidural analgesia may decrease the risk of cancer recurrence [71] and surgical site infection [72], although the published data supporting these effects is not yet convincing [73]. More controlled studies are needed to confirm these potentially exciting findings.

\section{Paravertebral blockade (PVB)}

Paravertabral blockades (PVB) have been used to achieve unilateral analgesia for surgical and traumatic processes in the chest and abdomen. Its analgesic capacity is compared to the gold standard for this setting, which is thoracic epidural analgesia, always at the expense of the administration of more volume and a greater concentration of LA although adverse effects such as hypotension, urinary retention and vomiting are much less. Its greatest inconvenience is the variable distribution of LA after the single injection technique, with a measure of four sensitive levels blocked after the initial recommended dose of $0.2-0.3 \mathrm{~mL} / \mathrm{kg}$ of $0.5 \%$ bupivacaine with adrenaline, as well as the time to the peak onset of action, which is $40 \mathrm{~min}$ and therefore it cannot be used as a preventive analgesia [74]. The failure rate for this technique is lower than that of the thoracic epidural and it is estimated to be above $6-10 \%$, although the use of a stimulator helps improve the success rate. A systematic review and meta-analysis [75] on 520 patients in which both techniques were compared reflected a similar anaesthetic quality with a better profile of adverse effects and pulmonary complications in favour of a paravertebral block. Moreover, it is advantageous in patients who receive anti-aggregation and are under general anaesthesia. Its advantages for use with video thoracoscopy have not been well demonstrated, but they have been demonstrated in breast surgery [76].

In a review by Scarci et al., [70] PVB was found to be of equal efficacy to epidural anaesthesia in patients undergoing thoracotomy surgery, but with a favourable side effect profile, and a lower complication rate. The reduced rate of complication was most marked for pulmonary complications and was accompanied by a quicker return to normal pulmonary function. The epidural block was associated with frequent side effects [urinary retention (42\%), nausea (22\%), itching (22\%) and hypotension (3\%) and, rarely, respiratory depression (0.07\%)]. Additionally, it prolonged operative time and was associated with technical failure or displacement $(8 \%)$. Epidurals were also related to a higher complication rate (atelectasis/pneumonia) compared to the PVB.

\section{Epidural coadjutants}

\subsection{Opioids}

The spinal administration of an opioid drug does not guarantee selective action and segmental analgesia in the spine. Evidence from experimental studies in animals indi- 
cates that bioavailability in the spinal cord biophase is negatively correlated with liposolubility, and is higher for hydrophilic opioids, such as morphine, than lipophilic opioids, such as fentanyl, sufentanil and alfentanil. All opioids administered produce part of their analgesic effect via spinal selectivity, although lipophilic opioids also rapidly reach higher centres of the brain due to their good vascular uptake and redistribution. Clinical trials have demonstrated that the administration of lipophilic opioids by continuous epidural infusion does not produce analgesia due to a spinal mechanism, nevertheless, by strengthening local anaesthesia they enable total doses to be reduced. This contrasts with single epidural injections of fentanyl, which with sufficiently high quantities of the drug can reach specific areas at the spinal level [77].

Morphine [78] is probably the opioid with the greatest medullary selective action after epidural (3-5 mg/day) or intradural administration. Morphine is the most used epidural opioid, and it could be considered the gold standard of spinal drugs (which does not imply it is the ideal one), because, due to its medullary selectivity, the epidural dose used is much lower than the parenteral dose (1/5-1/10), with a recommended daily maximum dose of $10 \mathrm{mg}$. It can be administered both in the form of boluses $(30-100 \mu \mathrm{g} / \mathrm{kg})$ and in a continuous infusion $(0,2-0$, $4 \mathrm{mg} / \mathrm{h}$ ), as the latter appears to induce a greater analgesic quality, and as a single drug or together with LAs, because these two drugs potentiate the global analgesic effect by means of a synergistic action, resulting in a postoperative analgesia of great quality and duration, but at the expense of a greater incidence of adverse effects. Despite epidural morphine being regarded as an effective drug via a route of administration that is just as effective, its use as a single dose is limited by its effective half-life of less than $24 \mathrm{~h}$, a short duration compared with that of postoperative pain. Liposomes are spherical particles formed by an external phospholipid layer and an internal aqueous chamber, where the drug is located. This is why in 2004, the FDA approved extended release epidural morphine (EREM) liposome injections only for lumbar epidural use, with a half-life of $48 \mathrm{~h}$ after a single injection, delaying the peak concentration in the CSF by up to $3 \mathrm{~h}$, without the problems associated with the catheter and with the expectation of improving the global failure rate by close to $30 \%$ of the continuous epidural technique. The basic points for its use include administration prior to surgery or after clamping the umbilical cord during a caesarean section and at least $15 \mathrm{~min}$. after the epidural test dose of LA and that no more epidural drugs be given for $48 \mathrm{~h}$, since the continuous infusion of LA increase the release of morphine. The formulation must not be injected through a filter as the particles may be disrupted [79]. As with all opioids, the chief hazard is respiratory depression especially in elderly and debilitated patients and in those with compromised respiratory function. In a meta-analysis on the risk of respiratory depression compared to intravenous morphine in patient-controlled analgesia (PCA), an odds ratio (OR) of 5.80 (95\% CI 1.05 - 31.93; $\mathrm{p}=0.04)$ was estimated for the use of EREM [80].

The continuous, solely epidural administration of fentanyl and sufentanil [77] offers very few advantages compared to its intravenous administration, which is why it is used with LAs to reduce its minimum effective analgesic concentration improving overall patient satisfaction. Lipophilic opioids such as fentanyl and sufentanil produce an analgesic effect mainly through systemic reuptake and their administration as a single drug does not offer any advantages 
compared to the parenteral route. However, their use with LAs enhances the analgesic effect, reducing the total dose of each of the drugs, as well as their adverse effects, such as hypotension and motor block. Fentanyl and sufentanil given epidurally or intradurally are the drugs of choice in obstetrics and ambulatory surgery, and are the coadjutants most commonly used together spinally with local anaesthetics in the perioperative period, improving analgesia without prolonging motor blockade. The spinal administration of alfentanil produces analgesia through systemic reuptake and redistribution to cerebral opioid receptors, as it has the greatest volume of distribution. Only fentanyl in bolus appears to present a specific medullary action in the group of lipophilic opioids in the epidural route at a concentration $>10 \mu \mathrm{g} / \mathrm{ml}$. Finally, [78] epidural methadone and hydromorphone are suitable alternatives for analgesia in the postoperative period, given that they have intermediate pharmacokinetic characteristics with respect to the two aforementioned groups of opioids.

\subsection{Other coadjutants}

The components of an ideal epidural solution for the control of postoperative pain are yet to be defined, as none achieves a total relief of the baseline pain at rest and of the breakthrough pain of a dynamic nature, without adverse effects such as hypotension, motor block, nausea, itching or sedation. However, from the studies published to date (clinical, randomized, controlled trials), we may draw the following conclusions with a high level of clinical evidence associated with the use of epidural adrenalin [81]:

- The combination of adrenalin with a mixture of low doses of bupivacaine $(0.1 \%)$ and fentanyl $(2 \mu \mathrm{g} / \mathrm{ml})$ has proven to be very effective in continuous infusion after major thoracoabdominal surgery, reducing the consumption of two other epidural drugs, as well as reducing their vascular absorption from the epidural space and improving the overall analgesic quality, efficacy and safety.

- The minimum analgesic concentration of adrenalin has been estimated to be $1.5 \mu \mathrm{g} / \mathrm{ml}$.

- Ropivacaine has proven to be equipotent to bupivacaine in the same epidural mix.

- The location of the epidural catheter must be metameric at the level of the thorax, as there is not enough scientific evidence to recommend the use of adrenalin in continuous infusion at the lumbar level.

Clonidine $(5-20 \mu \mathrm{g} / \mathrm{h})$ enhances the analgesic effect of the epidural mix, but the appearance of side effects such as hypotension, bradycardia or sedation limits its routine use. Neostigmine, a cholinesterase inhibitor, has been described as a strong analgesic coadjutant when using this route, at doses of $1-10 \mu \mathrm{g} / \mathrm{kg}$ after orthopaedic surgery to the knee, abdominal and gynaecological surgery, although it is limited by adverse effects such as sedation and nausea [82].

The objectives of a very recent quantitative systematic review were to assess both the analgesic efficacy and the safety of neuraxial magnesium. Eighteen published trials, comparing magnesium with placebos, have examined the use of neuraxial magnesium in its use as a perioperative adjunctive analgesic since 2002, with encouraging results. However, concurrent animal studies have reported clinical and histological evidence of neurological complications with 
similar weight-adjusted doses. The time to first analgesic request increased by $11.1 \%$ after intrathecal magnesium administration (mean difference: $39.6 \mathrm{~min}$; 95\% CI 16.3-63.0 min; $\mathrm{p}=0.0009$ ), and by $72.2 \%$ after epidural administration (mean difference: $109.5 \mathrm{~min} ; 95 \%$ CI 19.6-199.3 min; $\mathrm{p}=0.02$ ) with doses of between 50 and $100 \mathrm{mg}$. Four trials were monitored for neurological complications: of the 140 patients included, only a 4-day persistent headache was recorded. The authors concluded that despite promising perioperative analgesic effects, the risk of neurological complications resulting from neuraxial magnesium has not yet been adequately defined [83].

\section{Intradural opioid analgesia}

Intrathecal opioid administration can provide an excellent method of controlling acute postoperative pain and is an attractive analgesic technique since the drug is injected directly into the CSF, close to the structures of the central nervous system where the opioid acts. The procedure is simple, quick and has a relatively low risk of technical complications or failure. It is ever more frequent to associate opioids of different characteristics in the intradural route, a lipophilic opioid, such as fentanyl (20-40 $\mu \mathrm{g})$, and/or a hydrophilic opioid such as morphine (100-300 $\mu \mathrm{g})$, in the form of a bolus prior to surgery, together with LA, in order to guarantee coverage both during the immediate $(2-4 \mathrm{~h})$ and the late $(12-24 \mathrm{~h})$ postoperative period. Thus, associating a lipophilic opioid with bupivacaine or lidocaine leads to a shortening of the onset of the block and to an improvement of intraoperative analgesia as well as during the first hours of the postoperative period without prolonging the motor block or lengthening the time to discharge making it a good choice for ambulatory surgery [84].

In an excellent review by Rathmell JP et al. [85] on the use of intrathecal drugs in the treatment of acute pain, a maximum effective dose of morphine was advised, the negative effects of which seem to surpass the beneficial effects; after doses $>300 \mu \mathrm{g}$, nausea and itching usually appear, as well as severe urinary retention, and in studies on healthy volunteers, all of them presented with respiratory depression when the doses went beyond $600 \mu \mathrm{g}$.

In a meta-analysis [86] of 27 studies (15 concerning cardiothoracic, nine abdominal, and three spinal surgery) on a total of 645 patients who received doses between 100 and $4000 \mu g$, it was demonstrated that among those given intrathecal morphine VAS at rest, on a scale of $10 \mathrm{~cm}$, was $2 \mathrm{~cm}$ lower at $4 \mathrm{~h}$ and $1 \mathrm{~cm}$ lower at 12 and $24 \mathrm{~h}$, and this effect was more pronounced with movement, the relative improvement being more than $2 \mathrm{~cm}$ throughout the period of monitoring. This lower score on a VAS was significantly better than the outcome with other analgesic techniques such as the administration of IV ketamine at low doses (scores fell by $0.4 \mathrm{~cm}$ ), a regimen of postoperative NSAID (scores fell by $1 \mathrm{~cm}$ ), and even the continuous epidural infusion technique (scores fell by $1 \mathrm{~cm}$ ), as assessed by the same authors previously [87]. The doses of opioids required intra- and postoperatively up to $48 \mathrm{~h}$ were lower among those given intrathecal morphine and the use of morphine up to $24 \mathrm{~h}$ was significantly lower in the abdominal surgery group $(-24.2 \mathrm{mg}, \mathrm{CI}:-29.5$ to -19$)$ than the cardiothoracic surgery group $(-9.7 \mathrm{mg}$, CI: -17.6 to -1.80$)$. This more marginal benefit in the latter group makes the 
use of intrathecal morphine in thoracic surgery questionable, as a similar reduction in the amount of morphine required intravenously can be achieved using other strategies, such as the use of intraoperative ketamine $(-16 \mathrm{mg} / 24 \mathrm{~h}$ ) or postoperative NSAID ( -10 to $20 \mathrm{mg} / 24 \mathrm{~h}$ ) and even $4 \mathrm{mg}$ of IV paracetamol may be able to avoid using up to $8 \mathrm{mg}$ of morphine in the first day after surgery [88]. The adverse effects were indeed more common in the group given intrathecal morphine with an odds ratio of $7.8,3.8$ and 2.3 for respiratory depression, pruritus and urine retention, respectively, although interestingly there was not a higher rate of nausea or vomiting. Further, a recent meta-analysis has demonstrated that the addition of clonidine to intrathecal morphine extends the time to the first rescue analgesia in a postoperative setting by more than $75 \mathrm{~min}$. compared with morphine alone and it also reduces the amount of postoperative morphine by a mean of $4.45 \mathrm{mg}$ (95\% CI: 1.40-7.49). However, as the effects are small, and the results are heavily influenced by a study in which intrathecal fentanyl was also given, the authors concluded that this must be balanced with the increased frequency of hypotension [89].

Attempts have been made to define the optimal doses and drugs for a series of surgical procedures with the following recommendations [84-86]:

- Sufentanil 5-12.5 $\mu \mathrm{g}$, or fentanyl 10-25 $\mu \mathrm{g}$ for orthopaedic, ambulatory surgery and caesarean section, and fentanyl $5 \mu \mathrm{g}$ and sufentanil 2.5-5 $\mu \mathrm{g}$ for pain in labour, as sufentanil doses $>7.5$ $\mu \mathrm{g}$ are associated with foetal bradycardia.

- Morphine: 50-500 $\mu \mathrm{g}$ (Summarized in Figure n²)

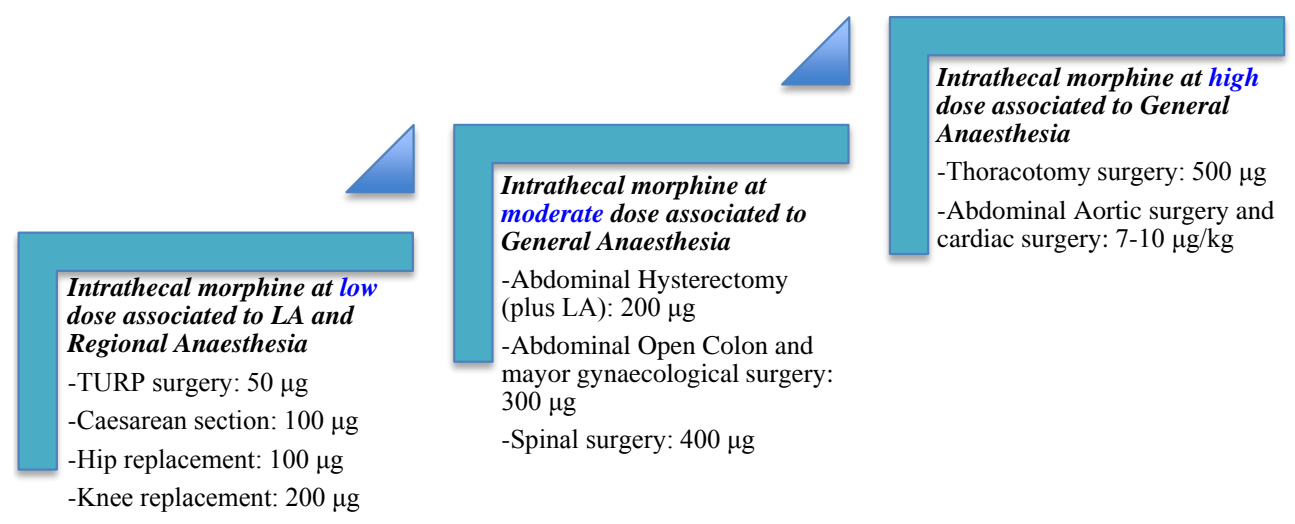

Figure 2. Recommended intrathecal morphine dosage for various surgical procedures in adults [84-86]

Key points for choosing the correct dose of intradural opioids [84-89]:

- Correct patient selection and minimum effective dose for each surgical procedure.

- Do not use morphine for ambulatory patients. Lyophilic opioids such as fentanyl and sufentanil are a better choice. 
- Morphine DOSES $\geq 300 \mu \mathrm{g} \rightarrow$ have an elevated risk of late respiratory depression 6-12 $h$.

- Morphine DOSES $<300 \mu \mathrm{g}$ have a similar risk to the parenteral administration of opioids.

- Monitored surveillance is recommended in the recovery or waking room or a mínimum monitoring for respiratory rate, oxygen levels (pulse oxymetry, if necessary) and above all, to monitor the level of consciousness for 12-24 h after intradural morphine and 4-6 h after fentanyl or sufentanil.

\section{Peri-incisional analgesia}

Peri-incisional analgesia is experiencing a great increase due to its ease of placement by the surgeon and its low profile of complications in the hospitalization ward (rate of infections < $0.7 \%$, without the systemic toxicity risk of LA). It is carried out using a multi-perforated catheter of a similar length to the surgical wound, with an infusion of a long action LA without a vasoconstrictor, in a variable location in the literature, but predominantly in a subcutaneous or subfascial location. It has advantages in a large variety of processes with incisions of 7 to $15 \mathrm{~cm}$ in length, with a lower VAS score, both at rest and in motion, as well as a lower consumption of opioids and a greater satisfaction for the patients, without affecting the hospital stay [16]. A systematic review, including 16 RCTs of patients undergoing major orthopaedic surgery and 15 RCTs undergoing cardiothoracic surgery, showed that postoperative pain management by wound catheter infusion was associated with decreased pain scores at rest and activity, opioid rescue dose, incidence of PONV and increased pain satisfaction [90]. However, a more recent meta-analysis was far less positive [91]. A total of 753 studies primarily fitted the search criteria and 163 were initially extracted. Of these, 32 studies were included in the meta-analysis. Wound catheters provided no significant analgesia at rest or during activity, except in patients undergoing gynaecological and obstetric surgery at $48 \mathrm{~h}(\mathrm{P}=0.03)$. The overall morphine consumption was lower $(\approx 13 \mathrm{mg})$ during $0-24 \mathrm{~h}(\mathrm{P}<0.001)$ in these patients. No significant differences in side effects were found, except for a lower risk of wound breakdown $(\mathrm{P}=0.048)$ and a shorter length of hospital stay $(\mathrm{P}=0.04)$ in patients receiving LA. Some authors disagree about these results arguing that these conclusions were due to the exclusion of orthopaedic patients and patients in whom catheters were not actually placed in the surgical wound [92].

A recent study has evaluated the efficacy of the preperitoneal continuous wound infusion (CWI) of ropivacaine for postoperative analgesia after open colorectal surgery in a multicentre randomized controlled trial. Over the 72-hour period after the end of surgery, CWI analgesia was not inferior to continuous epidural analgesia (CEA). The difference of the mean VAS score between CEI and CWI patients was $1.89(97.5 \%$ confidence interval $=-0.42,4.19)$ at rest and $2.76(97.5 \%$ confidence interval $=-2.28,7.80)$ after coughing. Secondary end points, morphine consumption and rescue analgesia, did not differ between groups. Time to first flatus was 3.06 \pm 0.77 days in the CWI group and $3.61 \pm 1.41$ days in the CEI group $(P=0.002)$. Time to first stool was shorter in the CWI than the CEI group $(4.49 \pm 0.99$ versus $5.29 \pm 1.62$ days; $\mathrm{P}=0.001)$. The mean time to hospital discharge was shorter in the CWI group than in the CEI group (7.4 
\pm 0.41 and $8.0 \pm 0.38$ days, respectively). More patients in the CWI group reported an excellent quality of postoperative pain control ( $45.3 \%$ versus $7.6 \%)$. The quality of night sleep was better with CWI analgesia, particularly at the postoperative 72 -hour evaluation $(\mathrm{P}=0.009)$. Postoperative nausea and vomiting were significantly less frequent with CWI analgesia at the 24 hours $(\mathrm{P}=0.02)$, 48 hours $(\mathrm{P}=0.01)$, and 72 hours $(\mathrm{P}=0.007)$ after surgery evaluations [93].

Appropriate catheter positioning is important, as it seems that preperitoneal placing is associated with better analgesia in patients undergoing open colorectal surgery, whilst subfascial placing provides good analgesia after caesarean section. The evidence-based PROSPECT recommendations include wound infiltration for inguinal herniotomy, laparoscopic cholecystectomy, hysterectomy, open colon surgery (preperitoneal infusion), total knee arthoplasty and haemorrhoidectomy [94]. This technique is also recommended by the ASA (American Society of Anesthesiology) practice guidelines as a part of a multimodal analgesia strategy for the management of postoperative pain [95].

\section{Evidenced-based clinical recommendations}

Due to the large variability of surgical interventions and the multiplicity of factors involved in postoperative pain, two initiatives have been put forward for drafting a practical guideline based on clinical evidence, specific for each process, and both are available on the Internet. One of them comes from the Veterans Health Administration of the US, in collaboration with the Defence Department and the University of Iowa (www.oqp.med.va.gov/cpg/cpg.htm), and the other from a working group of European anaesthesiologists and surgeons, the Prospect Working Group (www.postoppain.org). In the latter, the level of recommendation for each drug or medical acts for all of the perioperative periods are defined, and it currently contains 10 surgical procedures [94]. The Prospect Group helps physicians choose the most adequate drugs and technique combinations based on the published medical evidence and they are specialized in providing evidence-based and procedure-specific recommendations and clinical decision support for the management of postoperative pain. These are some examples for postoperative pain management:

This is the modus operandi of the Prospect Group:

1. Procedure-specific recommendations take into consideration the differences in character, location and severity of pain associated with different surgical procedures.

2. Evidence from a systematic review is supplemented with transferable evidence and expert knowledge from a Working Group of surgeons and anaesthesiologists.

3. Consensus recommendations are formulated by the Prospect Working Group, using established methods for group decision-making (Delphi method, Nominal Group Process).

4. Recommendations are graded to indicate the strength of recommendations (A-D). 
5. Recommendations are provided with an explanation of the evidence on which they are based, including the level (LoE 1-4) and source of evidence (procedure-specific or transferable).

6. All evidence from systematic reviews, as well as transferable evidence, is summarized and abstracts of all references are provided.

7. Studies included in the reviews are assessed and assigned a level of evidence: study design, quality, consistency and directness are taken into consideration.

8. Procedure-specific evidence, transferable evidence and clinical practice information (expert opinion) are clearly separated.

9. Benefits and harms of different interventions are indicated with a system of ticks and crosses, and the balance of benefits and harms is considered in formulating the recommendations.

10. Evidence and recommendations are freely accessible on the Internet at www.postoppain.org (Consult the original website for clarification of each level of recommendation)

- Recommendations for colonic surgery:

- Continuous thoracic epidural anaesthesia and analgesia at a level appropriate to the site of incision are recommended for routine use, based on superior postoperative analgesic and safety benefits compared with systemic techniques, if there is no contraindication for epidural administration. (Grade A)

- Where epidural techniques are used, it is recommended that a combination of strong opioid and LA must be used because of the increased analgesic efficacy compared with a strong opioid alone and to reduce the dose of opioids and their associated side effects. (Grade A)

- Preoperative administration of a single-shot epidural analgesia produces a similar postoperative analgesic efficacy to postoperative administration

- Continuous epidural anaesthesia and postoperative analgesia are recommended for routine use in colonic resection (Grade A), based on their benefits for reducing postoperative pain, systemic opioid use and improving bowel recovery time [(Level of evidence $1(\operatorname{LoE} 1)]$

- A combination of epidural local anaesthetic (LA) and strong opioid is recommended for epidural analgesia (Grade A), based on procedure-specific evidence of their combined efficacy, in reducing postoperative pain and systemic opioid use, compared with LA alone (LoE 1). However, the addition of opioid to epidural LA results in an increase in time to the first bowel movement. (LoE 1)

- Where epidural techniques are used, it is recommended that the epidural catheter be inserted preoperatively because this is the most practical timing for insertion. (Grade D, LoE 4) 
- COX-2-selective inhibitors (Grade B) (only for patients who do not receive epidural analgesia)

- Continuous administration of pre/intraoperative IV lidocaine if continued during the immediate postoperative period (Grade B), when epidural analgesia is not feasible or contra-indicated.

- Spinal analgesia is not recommended in combination with epidural anaesthesia (Grade B), based on the lack of benefit in reducing postoperative pain in colonic resection (LoE 2). Moreover, it introduces a greater level of complexity. (LoE 4)

- The decision concerning the type of operative technique or incision to use for colonic resection should be primarily based on factors other than the management of postoperative pain, e.g., malignancy versus benign disease operative risk factors of the patient, risk of wound infection, and availability of surgical expertise (Grade D)

- Laparoscopic colonic resection is recommended over open colon surgery for reducing postoperative pain, if the conditions outlined above allow (Grade A)

- A horizontal/curved (transverse) incision is recommended over a vertical incision for analgesic and other benefits if the operative conditions allow (Grade B). In addition, the horizontal/curved incision is preferred for its cosmetic benefits (Grade D)

- Diathermy is recommended over the scalpel (Grade C)

- Maintenance of normothermia is recommended for improved clinical outcomes, but it is not helpful for reducing postoperative pain (Grade A)

- Postoperative Recommended Systemic Analgesia:

- COX-2-selective inhibitors (Grade B) (only for patients who are not receiving epidural analgesia or upon the cessation of epidural analgesia)

- Conventional NSAIDs (Grade A) (only for patients who are not receiving epidural analgesia or upon the cessation of epidural analgesia)

- IV lidocaine (Grade B) (when epidural is not feasible or contra-indicated)

- Strong opioids (Grade B) (for high-intensity pain)

- Weak opioids (Grade B) in association with other non-opioid analgesics (for moderateor low-intensity pain), or if non-opioid analgesia is insufficient or contra-indicated

- Paracetamol (Grade B) for moderate- or low-intensity pain (only for patients who do not receive epidural analgesia, or after the cessation of epidural analgesia)

\section{- Recommendations for post-thoracotomy pain:}

- Pre- and intraoperative thoracic epidural or Paravertebral Blockade (PVB) are recommended based on the reduction in pain compared with postoperative administration alone. (Grade A) 
- PVB LA or thoracic epidural LA plus a strong opioid is recommended as a preoperative bolus followed by an infusion continued for 2-3 days postoperatively, based on a reduction in pain compared with systemic analgesia. (Grade A)

- There are not enough data to recommend one specific combination of LA over another, or a specific concentration or volume.

- There are not enough data to recommend lipophilic opioids in preference to hydrophilic opioids or vice versa, in combination with LA.

- Thoracic epidural LA plus an opioid is recommended in preference to a spinal strong opioid based on evidence that the analgesic effect of thoracic epidural analgesia has a longer duration than $24 \mathrm{~h}$. (Grade A)

- A preoperative single bolus of a spinal strong opioid is recommended as part of a multianalgesic regimen (Grade A), when epidural analgesia or paravertebral blocks are not possible for any reason (Grade D). Repeated perioperative doses via the spinal route are not recommended because they are not considered to be safe or practical. (Grade D)

- Spinal opioids are recommended in preference to intravenous PCA opioids, based on a greater reduction in pain for up to 24 hours, with no difference in respiratory function. (Grade A)

- Lumbar epidural strong opioid is not recommended as the first choice based on evidence that the thoracic epidural route is more effective for pain relief (Grade A). However, there is procedure specific evidence that lumbar hydrophilic strong opioid reduces pain compared with systemic analgesia.

- Epidural epinephrine is recommended if a low dose of epidural LA and/or opioid is used (Grade B).

- Intercostal nerve block with LA (bolus at the end of surgery, followed by continuous infusion), if thoracic epidural analgesia and paravertebral blocks are not possible (Grade D)

- Postoperative Recommended Systemic analgesia:

- Conventional NSAIDs, if regional analgesia is inadequate (Grade A)

- COX-2-selective inhibitors, if regional analgesia is inadequate (Grade B)

- Intravenous PCA strong opioid, if regional analgesic techniques fail or are not possible (Grade D)

- Weak opioids for moderate- (VAS $>30<50 \mathrm{~mm}$ ) or low- (VAS $<30 \mathrm{~mm}$ ) intensity pain in the late postoperative period, only if conventional NSAIDs/COX-2-selective inhibitors plus paracetamol are insufficient or contra-indicated (Grade D)

- Paracetamol, if regional analgesia is inadequate, as part of a multianalgesic regimen (Grade D) 
- Recommendations for Abdominal Hysterectomy:

- General anaesthesia, or single dose spinal anaesthesia with or without light general anaesthesia in low-risk patients (grade D)

- Epidural anaesthesia combined with light general anaesthesia or combined spinalepidural anaesthesia, in high-risk patients (grade A)

- Strong opioids administered in time to secure sufficient analgesia when the patient wakes up (grade A)

-Wound infiltration before closure (grade A)

- LAVH or VH rather than abdominal hysterectomy, only if allowed by the surgical requirements (based on technical feasibility, patient indication for hysterectomy and risk factors) (grade A)

- Pfannenstiel incision, only if allowed by the surgical requirements (based on technical feasibility, patient indication for hysterectomy and risk factors) (grade B)

- Diathermy incision (grade B)

- Active patient warming in high-risk patients (grade A)

- Intraoperative music (grade A)

- Postoperative Recommended Systemic Analgesia:

- COX-2 selective inhibitors or conventional NSAIDs, in combination with strong opioids for high-intensity pain (VAS $>50 \mathrm{~mm}$ ) or with weak opioids for moderate- (VAS $<50>30$ ) or low-intensity pain (VAS $<30 \mathrm{~mm}$ ) (grade A)

- Strong opioids via IV PCA or via fixed IV dosing titrated to pain intensity (grade A)

- Paracetamol for moderate- (VAS $>30<50$ ) or low-intensity (VAS $<30 \mathrm{~mm}$ ) pain, in combination with COX-2 inhibitors or conventional NSAIDs (grade A)

- Recommendations for total hip arthroplasty:

- COX-2-selective inhibitors or conventional NSAIDs (grade A) in combination with paracetamol and/or strong opioids for high-intensity pain (grade A) or with paracetamol and/or weak opioids for moderate- or low-intensity pain (grade D)

- Strong opioids in combination with non-opioid analgesia to manage high-intensity pain (grade A), in time to provide analgesia in the early postoperative recovery period, administered by IV patient-controlled analgesia (grade A) or IV titrated for pain intensity (grade D)

- Weak opioids for moderate- or low-intensity pain if conventional NSAIDs or COX-2selective inhibitors are insufficient or are contra-indicated (grade D)

- Paracetamol (grade A) in combination with conventional NSAIDs or COX-2-selective inhibitors, with or without rescue opioids (grade B) 
- Epidural infusion with local anaesthetic plus opioid for cardiopulmonary risk patients (grade B), in time to provide analgesia in the early postoperative recovery period (grade D)

- Posterior lumbar plexus block (psoas sheath blocks) (grade A) or femoral nerve block (grade B) or single-bolus spinal morphine as a part of spinal anaesthesia (grade B), depending on the balance of efficacy and risks for the individual patient

- Intraoperative, high-volume, low-concentration wound infiltration (LIA) (grade A)

- Recommendations for total knee arthroplasty:

- Pre or postoperative Femoral nerve block is recommended (Grade A) based on evidence of a reduction in pain scores and supplemental analgesia (procedure-specific evidence, LoE 1)

- No recommendation can be made concerning continuous femoral infusion techniques versus a single bolus because of the heterogeneity in the study design and the inconsistency of procedure-specific data (LoE 4).

- Spinal LA + opioid is recommended (Grade A, LoE 1), but not as the first choice of analgesic technique because of a greater potential for adverse events compared with femoral nerve block (transferable evidence, LoE 3)

- Morphine is recommended as the opioid in the spinal LA + opioid combination (Grade A) based on evidence for a longer duration of analgesic effect than other opioids (procedure-specific evidence, LoE 1)

- Preoperative epidural analgesia (LA and/or opioid) is not recommended as the first choice but it can be used if a femoral blockade is not possible (Grade B).

There is also overall scientific evidence published on the treatment of APP, which is summarized in figure $\mathrm{n}^{\circ} 3$ [97]. In the case of ambulatory surgery, [98] multimodal or balanced regimens of analgesia based on non-opioid drugs have been imposed in order to reduce adverse effects such as nausea and/or vomiting. Moreover, preventive analgesia has been promoted which aims to achieve better control of postoperative pain, as it is one of the most important factors for readmission. It has been proven that a combined regimen of dexamethasone at a single preoperative dose, incision LA (at the beginning or at the end of the surgery) and a postoperative regimen of 3-5 days of NSAIDs (COXIB or non-selective NSAIDs) achieved the best results in the control of pain and in the reduction of the time of convalescence. The association of paracetamol, gabapentinoids and the continuous infusion of peri-incisional LA in an ambulatory setting have also achieved a beneficial effect in patients. In the case of a poor control of pain, opioid rescue medication, such as tramadol or oral oxycodone could be necessary.

(Ia) meta-analysis, including at least one controlled and randomized study with a large number of cases, (Ib) the same, but with fewer cases, (II) well designed cohort or case-control studies, (III) well designed descriptive, non-experimental studies (IV) studies based on expert opinions or committees, (V) insufficient evidence to reach an opinion. 
Evidence Level Ia

-IV PCA provides a better analgesia than parenteral opioids administered by the nursing staff

- The techniques for regional, peripheral, continuous analgesia provide better analgesia than systemic opioids - Multimodal analgesia (multidoses of NSAIDs, COX-2

inhibitors, or paracetamol and IV-PCA with opioids) improves pain control and reduces the adverse effects of opioids

- Continuous epidural analgesia is more beneficial after major surgery (< morbidity, < paralytic ileus and > ability to walk) than parenteral opioids in patients with cardiopulmonary disease
Evidence Level Ib

- The creation of practical guidelines for managing APP has improved the approach of APP - Opioid-sparing regimens reduce recovery time of bowel function after abdominal surgery
Evidence level II-III -Poor APP control predisposes to developing chronic, postsurgical pain (EL II)

-APP must be strongly controlled in chronic patients with opioid tolerance (EL III)

Figure 3. Analgesic strategies with the Evidence Level (EL) in APP [97]:

\section{Combination of drugs and rehabilitation programme in surgical patients}

It is normal daily practice to combine analgesics in order to improve the overall quality and patient satisfaction, but this does not mean we always meet our goal. Based on the studies that included controlled clinical trials or systematic reviews, that compare one drug with a combination of the same drug with one or more additional drugs via the same route of administration, Curatolo $\mathrm{M}$ et al. obtained the conclusions summarized in table IV [96].

The data currently available show that a multimodal programme of postoperative physical therapy and rehabilitation [99] can reduce the length of hospital stay, improve the control of dynamic pain and reduce the morbidity and mortality associated with the surgical procedure. We must begin with postoperative care that includes pain as the fifth vital sign, the use of regional analgesia to decrease opioid consumption, a responsible fluid therapy, maintaining normal body temperature, early mobilization, shortening the return to oral intake, avoiding motionrestriction factors such as drains, as well as improving postoperative sleep and stress, as they play a key role in reducing convalescence. This has led to the creation of ambulatory surgery units requiring coordination between all the healthcare specialists involved. Acute postoperative pain units are the key starting point for setting these programmes into motion.

Among the variety of surgical procedures, the recovery programme for colorectal surgery is one of the most studied and evaluated in the last decade. A recent meta-analysis concluded that the implementation of four or more elements of the Enhance Recovery After Surgery (ERAS) pathway leads to a reduction in the length of hospital stay by more than two days and an almost $50 \%$ reduction in complication rates in patients undergoing major colonic/colorectal surgery [100]. However, on the other hand, a Cochrane review of fast track surgery versus conventional recovery strategies for colorectal surgery concluded that the quality of the trials 
and the lack of other sufficient outcomes parameters do not justify the implementation of fasttrack surgery as the standard for care [101].

\section{Drug Combination}

$\square$ Adding NSAIDs to opioids

Adding paracetamol to opioids

Associating paracetamol + opioids

$\square$ Adding a weak opioid to paracetamol

Adding a weak opioid to an NSAID

Adding IV ketamine to an opioid

Adding an epidural opioid to the LA

Adding clonidine to the epidural mix

Adding adrenalin to the epidural mix

\section{Efficacy in Acute Postoperative Pain (APP)}

Improved analgesia and less side effects

Improved analgesia and less side effects

Better than each one separately

Questionable usefulness in minor surgery

Questionable usefulness in minor surgery

Probable usefulness $\rightarrow$

Monitor the narrow therapeutic range

Useful

There is no clear benefit

Useful in thoracic epidural analgesia

Table 4. Efficacy of pharmacological combination in acute postoperative pain (APP) [96]

\section{Discussion}

In 2007, a review was published on the clinical evidence of the effect of postoperative analgesia on the major postoperative complications with the following conclusions [102]: the positive effects of epidural analgesia on cardiovascular events or on lung function are limited to highrisk patients or to major vascular surgery, which, in some cases, is irrelevant when using an endovascular technique, and those that are beneficial in the presence of paralytic ileus can be minimized by laparoscopic techniques and fast-track programmes. Moreover, they found no evidence that the perineural or peri-incisional administration of LA, the administration of opioids by PCA, or the programmes of postoperative multimodal analgesia had any positive beneficial effects on postoperative complications, although they do improve overall patient satisfaction.

Indeed, many authors have questioned the use of epidural analgesia as the first choice of technique in the recovery protocols after mayor surgery. Rawal N. [103] thinks that epidural analgesia is a well-established technique that has commonly been regarded as the gold standard in postoperative pain management. However, newer, evidence-based outcome data 
show that the benefits of epidural analgesia are not as significant as previously believed, and that there are some benefits by decreasing the incidence of cardiovascular and pulmonary complications, but these benefits are probably limited to high-risk patients undergoing major abdominal or thoracic surgery who receive thoracic epidural analgesia with local anaesthetic drugs only. In the review, it was demonstrated that there is increasing evidence that less invasive regional analgesic techniques are as effective as epidural analgesia. These include paravertebral block for thoracotomy, femoral block for total hip and knee arthroplasty, wound catheter infusions for caesarean delivery and colon surgery, and local infiltration analgesia techniques for lower limb joint arthroplasty. Wound infiltration techniques and their modifications are simple and safe alternatives for a variety of other surgical procedures. The author also argues that although pain relief associated with epidural analgesia can be outstanding, clinicians expect more from this invasive, high-cost, labour-intensive technique and that the number of indications for the use of epidural analgesia seems to be decreasing for a variety of reasons. The main conclusion is that the decision about whether to continue using epidural techniques should be guided by regular institutional audits and careful risk-benefit assessment rather than by tradition.

Finally, practice guidelines for acute postoperative pain management have been recently published. The experts recommend anaesthesiologists who manage perioperative pain to use therapeutic options such as epidural or intrathecal opioids, systemic opioid PCA, and regional techniques after thoughtfully considering the risks and benefits for the individual patient. These modalities should be used in preference to IM opioids ordered "as needed". Consultants and ASA members also strongly agree that the therapy selected should reflect the individual anaesthesiologist's expertise, as well as the capacity for the safe application of the modality in each practiced setting. Special caution should be taken when continuous infusion modalities are used, as drug accumulation may contribute to adverse events. [95]

\section{Conclusions}

Although great work is being carried out in the area of postoperative pain, there is still a long way to go. It is necessary to apply a multimodal approach to pain that includes the routine use of regional techniques, a combination of analgesics such as paracetamol, non-specific or COX-2 NSAIDs and opioids by different routes, making a responsible choice for the type of patient, the surgical management and the predicted adverse effects. The true role of coadjutant drugs and non-pharmacological therapies is yet to be seen, and in the future, it will be essential to have a practical guide based on clinical evidence for each process, that includes postsurgical rehabilitation.

We must delve into the pathophysiology of pain, and in the direct application of this knowledge to new drugs and new systems for drugs delivery that achieve a lower number of postoperative complications, as well as a better overall recovery and general well-being of the patients. Healthcare professionals must be trained in the field of pain and their work must be coordinated within an acute postoperative pain unit, the structure of which must be stable and 
multidisciplinary, so as to arrive at agreed analgesic regimens with surgical and nursing departments. In the future, the goal must be to also cover the late postoperative period with the creation of postsurgical acute and chronic pain units.

\section{Author details}

Borja Mugabure Bujedo, Silvia González Santos, Amaia Uría Azpiazu, Anxo Rubín Noriega, David García Salazar and Manuel Azkona Andueza

Department of Anaesthesiology, Critical Care and Pain Medicine, Donostia University Hospital, San Sebastian, Spain

\section{References}

[1] Boswell MB, Giordano J. Reflection, analysis and change: the decade of pain control and research and its lessons for the future of pain management. Pain Physician 2009; 12(6) 923-928.

[2] Heitz JW, Witkowski T, Viscusi ER. New and emerging analgesics and analgesic technologies for acute pain management. Current Opinion in Anesthesiology 2009; 22(5): 608-617

[3] Shang AB, Gan TJ. Optimizing postoperative pain management in the ambulatory patient. Drugs 2003; 63(9) 855-867

[4] Cousins MJ, Brennan F, Car DB. Pain relief: a universal human right. Pain 2004; 112(1-2) $1-4$

[5] Brown AK, Christo PJ, Wu CL. Strategies for postoperative pain management. Best Practice \& Research Clinical Anesthesiology 2004; 18(4) 703-717

[6] Joshi GP, Ogunnaike BO. Consequences of inadequate postoperative pain relief and chronic persistent postoperative pain. Anesthesiology Clinics of North America 2005; 23(1) 21-36

[7] Perkins FM, Kehlet H. Chronic pain as an outcome of surgery. A review of predictive factors. Anesthesiology 2004; 93(4) 1123-1133

[8] Kehet H, Jensen TS, Woolf CJ. Persistent postsurgical pain: risk factors and prevention. Lancet 2006; 13; 367(9522) 1618-1625

[9] Clarke H, Bonin RP, Orser BA, Englesakis M, Wijeysundera DN, Katz J. The Prevention of Chronic Postsurgical Pain Using Gabapentin and Pregabalin: A Combined Systematic Review and Meta-Analysis. Anesthesia \& Analgesia 2012; 115(2) 428-442 
[10] Ip HY, Abrishami A, Peng PW, Wong J, Chung F. Predictors of postoperative pain and analgesic consumption: a qualitative systematic review. Anesthesiology 2009; 111(3) 657-677

[11] Ong CK, Lirk P, Seymour RA, Jenkins BJ. The efficacy of preemptive analgesia for acute postoperative pain management: a meta-analysis. Anesthesia \& Analgesia 2005; 100(3) 757-73,

[12] Moiniche S, Kehlet H, Dahl JB. A qualitative and quantitative systematic review of preemptive analgesia for postoperative pain relief: the role of timing of analgesia. Anesthesiology 2002; 96(3) 725-41.

[13] Koppert W, Schmelz M. The impact of opioid induced hyperalgesia for postoperative pain. Best Practice \& Research Clinical Anesthesiology 2007; 21(1) 65-83

[14] Wilder-Smith OH, Arendt-Nielsen L. Postoperative Hyperalgesia: its clinical importance and relevance. Anesthesiology 2006; 104(3) 601-607

[15] Lavand'homme P. Perioperative pain. Current Opinion in Anaesthesiology 2006; 19(5) 556-561

[16] Mugabure Bujedo B, Tranque Bizueta I, González Santos S, Adrián Garde R. Multimodal approaches to postoperative pain management and convalescence. Revista de la Sociedad Española de Anestesiología 2007; 54(1) 29-40

[17] Gajraj NM, Joshi GP. Role of cyclooxygenase-2 inhibitors in postoperative pain management. Anesthesiology Clinics of North America 2005; 23(1) 49-72

[18] Kaye AD, Baluch A, Kaye AJ, Ralf G, Lubarsky D. Pharmacology of cyclooxygenase-2 inhibitors and preemptive analgesia in acute pain management. Current Opinion in Anesthesiology 2008; 21(4) 439-445

[19] Caldwell B, Aldington S, Weartherall M, Schirtcliffe P, Beasley R. Risk of cardiovascular events and celecoxib: a systematic review and meta-analysis. Journal of the Royal Society of Medicine 2006; 99(3) 132-140

[20] Hernández-Díaz S, Varas-Lorenzo C, García Rodríguez LA. Non-steroidal antiinflammatory drugs and the risk of acute myocardial infarction. Basic \& Clinical Pharmacology \& Toxicology 2006; 98(3) 266-274

[21] Clarke R, Derry S, Moore RA.. Single dose oral etoricoxib for acute postoperative pain in adults. The Cochrane Database of Systematic Reviews 2012; 18(4) CD004309

[22] Kranke P, Morin AM, Roewer N, Eberhart LH. Patients' global evaluation of analgesia and safety of injected parecoxib for postoperative pain: a quantitative systematic review. Anesthesia \& Analgesia 2004; 99(3) 797-806,

[23] Remy C, Marret E, Bonnet F. State of the art of paracetamol in acute pain therapy. Current Opinion in Anesthesiology 2006; 19(5) 562-565 
[24] Remy C, Marret E, Bonnet F. Effects of acetaminophen on morphine side effects and consumption after major surgery: meta-analysis of randomized controlled trials. British Journal of Anaesthesia 2005; 94(4) 505-513

[25] Ong KS, Seymour RA, Lirk P, Merry AF. Combining paracetamol with NSAIDs: A qualitative systematic review of analgesic efficacy for acute postoperative pain. Anesthesia \& Analgesia 2010; 110(4) 1170-1179

[26] Miranda HF, Puig MM, Prieto JC, Pinardi G. Synergism between paracetamol and nonsteroidal anti-inflammatory drugs in experimental acute pain. Drugs 2006; 121(1-2) $22-28$

[27] Edwards J, Meseguer F, Faura C, Moore RA, McQuay HJ, Derry S. Single dose dipyrone for acute postoperative pain. Cochrane Database of Systematic Reviews 2010; 8(9) CD003227

[28] Derry P, Derry S, Moore RA, McQuay HJ. Single dose oral diclofenac for acute postoperative pain in adults. Cochrane Database of Systematic Reviews 2009; 15(2) CD004768

[29] Gan TJ, Daniels SE, Singla N, Hamilton DA, Carr DB. A Novel Injectable Formulation of Diclofenac Compared with Intravenous Ketorolac or Placebo for Acute Moderateto-Severe Pain After Abdominal or Pelvic Surgery: A Multicenter, Double Blind, Randomized, Multiple-Dose Study. Anesthesia \& Analgesia 2012; 115(5) 1212-1220

[30] Barden J, Derry S, Moore RA, McQuay HJ. Single dose oral ketoprofen and dexketoprofen for acute postoperative pain in adults. Cochrane Database of Systematic Reviews 2009; 7(4) CD007355

[31] Smith LA, Carroll D, Edwards JE, Moore RA, McQuay HJ. Single doses ketorolac and pethidine in acute postoperative pain: systematic review with meta-analysis. British Journal of Anaesthesia 2000; 84(1) 48-58

[32] Forrest JB, Camu F, Greer IA, H Kehlet, Abdalla M, Bonnet F, et al. Ketorolac, diclofenac and ketoprofen are equally safe for pain relief after major surgery. British Journal of Anaesthesia 2002; 88(2) 227-233,

[33] Moore RA, Derry S, McQuay HJ, Wiffen PJ. Single dose analgesics for acute postoperative pain in adults. Cochrane Database of Systematic Reviews 2011; 7(9) CD008659

[34] Guindon J, Walczak JS, Beaulieu P. Recent advances in the pharmacological management of pain. Drugs 2007; 67(15) 2121-2133

[35] Grass JA. Patient-controlled analgesia. Anesthesia \& Analgesia 2005; 101(5 Suppl) 44-61

[36] Scott LJ, Perry CM. Tramadol: a review of its use in perioperative pain. Drugs 2000; 60(1) $139-176$ 
[37] Lugo RA, Kern SE. The pharmacokinetics of oxycodone. Journal of Pain \& Palliative Care Pharmacotherapy 2004; 18(4) 17-30

[38] Kokki H, Kokki M, Sjövall S. Oxycodone for the treatment of postoperative pain. Expert Opinion on Pharmacotherapy 2012; 13(7) 1044-1058

[39] Nossaman VE, Ramadhyani U, Kadowitz PJ, Nossaman BD. Advances in perioperative pain management: use of medications with dual analgesic mechanisms, tramadol \& tapentadol. Anesthesiology Clinics 2010; 28(4) 647-666

[40] C.T. Hartrick. Tapentadol immediate release for the relief of moderate to severe acute pain. Expert Opinion on Pharmacotherapy 2009; 10(16) 2687-2696

[41] Vadivelu N, Mitra S, Narayan D. Recent advances in postoperative pain management. The Yale Journal of Biology and Medicine 2010; 83(1) 11-25

[42] Bell RF, Dahl JB, Moore RA, Kalso A. Perioperative ketamine for acute postoperative pain. Cochrane Database of Systematic Reviews 2006; 25(1) CD004603

[43] Laskowski K, Stirling A, McKay WP, Lim HJ. A systematic review of intravenous ketamine for postoperative analgesia. Canadian Journal of Anaesthesia 2011; 58(10) 911-923

[44] Blaudszun G, Lysakowski C, Elia N, Tramer MR. Effect of perioperative systemic $\alpha-2$ agonists on postoperative morphine consumption and pain intensity: systematic review and meta-analysis of randomized controlled trials. Anesthesiology 2012; 116(6) 1312-1322

[45] Dauri M, Faria S, Gatti A, Celedonio L, Carpenedo R, Sabato AF. Gapapentin and pregabalin for the acute postoperative pain management. A systematic-narrative review of the recent clinical evidences. Current Drugs Targets 2009; 10(8) 716-733

[46] Engelman E, Cateloy F. Efficacy and safety of perioperative pregabalin for postoperative pain: a meta-analysis of randomized-controlled trials. Acta Anaesthesiologica Scandinavica 2011; 55(8) 927-943

[47] Wang B, He KH, Jiang MB, Liu C, Min S. Effect of prophylactic dexamethasone on nausea and vomiting after laparoscopic gynecological operation: meta-analysis. Middle East Journal of Anesthesiology 2011; 21(3) 397-402

[48] Rawlinson A, Kitchingham N, Hart C, McMahon G, Ong SL, Khanna A. Mechanism of reducing postoperative pain, nausea and vomiting: a systematic review of current techniques. Evidence-Based Medicine 2012; 17(3) 75-80

[49] Schaub I, Lysakowski C, Elia N, Tramér MR. Low-dose droperidol ( $\leq 1 \mathrm{mg}$ or $\leq 15 \mu \mathrm{g} /$ $\mathrm{kg}-1$ ) for the prevention of postoperative nausea and vomiting in adults: quantitative systematic review of randomized controlled trials. European Journal of Anaesthesiology 2012; 29(6) 286-294 
[50] Vigneault L, Turgeon AF, Coté D, Lauzier F, Zarychanski R, Moore L, et al. Perioperative intravenous lidocaine for postoperative pain control: a meta-analysis of randomized controlled trials. Canadian Journal of Anesthesia 2010; 58(1) 22-37

[51] McCarthy CG, Megalla SA, Habib AS. Impact of intravenous lidocaine infusion on postoperative analgesia and recovery from surgery: a systematic review of randomized controlled trials. Drugs 2010; 70(9) 1149-1163

[52] De Oliveira GS Jr, Fitzgerald P, Streicher LF, Marcus RJ, McCarthy RJ. Systemic lidocaine to improve postoperative quality of recovery after ambulatory laparoscopic surgery. Anesthesia \& Analgesia 2012; 115(2) 262-267

[53] Albrecht E, Kirkham KR, Liu SS, Brull R. Peri-operative intravenous administration of magnesium sulphate and postoperative pain: a meta-analysis. Anaesthesia 2013; 68(1) 79-90

[54] White PF. The changing role of non-opioid analgesic techniques in the management of postoperative pain. Anesthesia \& Analgesia 2005; 101(5 Suppl) 5-22

[55] Aubrun F, Mazoit JX, Riou B. Postoperative intravenous morphine titration. British Journal of Anaesthesia 2012; 108(2) 193-201

[56] Mayes S, Ferrone M. Fentanyl HCI patient-controlled iontophoretic transdermal system for the management of acute postoperative pain. The Annals of Pharmacotherapy 2006; 40(12) 2178-2186

[57] Añez Simón C, Rull Bartomeu M, Rodríguez Pérez A, Fuentes Baena A. Intranasal opioids for acute pain. Revista de la Sociedad Española de Anestesiología 2006; 53(10) 643-652

[58] Viscusi ER. Patient-controlled drug delivery for acute postoperative pain management: a review of current and emergency technologies. Regional Anesthesia and Pain Medicine 2008; 33(2) 146-158

[59] Richman JM, Liu SS, Courpas G, Wong R, Rowlingson AJ, McGready J et al. Does continuous peripheral nerve block provide superior pain control to opioids? A Metaanalysis. Anesthesia \& Analgesia 2006; 102(1) 248-257

[60] Handley GH, Silbert BS, Mooney PH, Schweitzer SA, Allen NB. Combined general and epidural anaesthesia versus general anaesthesia for major abdominal surgery: post anesthesia recovery characteristics. Regional Anesthesia and Pain Medicine 1997; 22(5) 435-441

[61] Wheatley RG, Schug SA, Watson D. Safety and efficacy of postoperative epidural analgesia. British Journal of Anaesthesia 2001; 87(1) 47-61

[62] Wigfull J, Welchew E. Survey of 1057 patients receiving postoperative patientcontrolled epidural analgesia. Anaesthesia 2001; 56(1) 70-75 
[63] Block BM, Liu SS, Rowlingston AJ, Cowan AR, Cowan JA Jr, Wu CL. Efficacy of postoperative epidural analgesia: A meta-analysis. The Journal of the American Medical Association 2003; 290(18) 2455-2463

[64] Rodgers A, Walker N, Schug S, McKee A, Kehlet H, Van Zundet A, et al. Reduction of postoperative mortality and mobility with epidural or spinal anaesthesia: results from overview of randomized trial. British Medical Association 2000; 321(7275) 1493-1496

[65] Peyton PJ, Myles PS, Silbert BS, Rigg JA, Jamrozik K, Parsons R. Perioperative epidural analgesia and outcome after major abdominal surgery in high-risk patients. Anesthesia \& Analgesia 2003; 96(2) 548-554

[66] Rigg JR, Jamrozik K, Myles PS, Silbert BS, Peyton PJ, Parsons R, Collins KS., MASTER Anaesthesia Trial Study Group: Epidural Anaesthesia and analgesia and outcome of major surgery: a randomized trial. Lancet 2002; 359(9314) 1276-1282

[67] Hermanides J, Hollmann MW, Stevens MF, Lirk P. Failed epidural: causes and management. British Journal of Anaesthesia 2012; 109(2) 144-154

[68] Kehlet H. Procedure-specific postoperative pain management. Anesthesiology Clinics of North America 2005; 23(1) 203-210

[69] Low J, Jonhnston N, Morris C. Epidural analgesia: First do no harm. Anaesthesia 2008; 63(1) 1-3

[70] Scarci M, Joshi A, Attia R. In patients undergoing thoracic surgery is paravertebral as effective as epidural analgesia for pain management? Interactive Cardiovascular Thoracic Surgery 2010; 10(1) 92-96

[71] Yeager MP, Rosenkranz KM. Cancer recurrence after surgery: A role for regional anaesthesia. Regional Anesthesia and Pain Medicine 2010; 35(6) 483-484

[72] Chang CC, Lin HC, HW Lin, Lin HC. Anesthetic management and surgical site infections in total hip and knee replacement: A population-based study. Anesthesiology 2010; 113(2) 279-284

[73] Tsui BCH, Green JS. Type of anaesthesia during cancer surgery and cancer recurrence. British Medical Journal 2011;342: d1605

[74] Cheema S, Richardson J, McGurgan P. Factors affecting the spread of bupivacaine in the adult thoracic paravertebral space. Anaesthesia 2003; 58(7) 684-687.

[75] Davies RG, Myles PS, Graham JM. A comparison of the analgesic efficacy and side effects of paravertebral vs. epidural blockade for thoracotomy: A systematic review and meta-analysis of randomized trials. British Journal of Anaesthesia 2006; 96(4) 418-26

[76] Schnabel A, Reichl SU, Kranke P, Pogatzki-Zahn EM, Zahn PK. Efficacy and safety of paravertebral blocks in breast-surgery: a meta-analysis of randomized trials. British Journal of Anaesthesia 2010; 105(6) 842-852 
[77] Bernards CM. Recent insights into the pharmacokinetics of spinal opioids and the relevance to opioid selection. Current Opinion in Anaesthesiology 2004; 17(5) 441-447

[78] Bujedo BM, Santos SG, Azpiazu AU. A review of epidural and intrathecal opioids used in the management of postoperative pain. Journal of Opioid Management 2012; 8(3) 177-192

[79] Hartrick CT, Hartrick KA. Extended-released epidural morphine (Depodur ${ }^{\mathrm{TM}}$ ): review and safety analysis. Expert Review of Neurotherapeutics 2008; 8(11) 1641-1648,

[80] Sumida S, Lesley MR, Hanna MN, Murphy JD, Kumar K, Wu CL. Meta-analysis of the effect of extended-release epidural morphine versus intravenous patient-controlled analgesia on respiratory depression. Journal of Opioid Management 2009; 5(5) 301-305

[81] Niemi G. Advantages and disadvantages of adrenaline in regional anaesthesia. Best Practice \& Research Clinical Anaesthesiology 2005; 19(2) 229-245

[82] Congedo E, Sgreccia M, De Cosmo G. New Drugs for epidural analgesia. Current Drug Targets 2009; 10(8) 696-706

[83] Albrecht E, Kirkham KR, Liu SS, Brull R. The analgesic efficacy and safety of neuraxial magnesium sulphate: a quantitative review. Anaesthesia 2013; 68(2): 190-202.

[84] Mugabure Bujedo B. A clinical approach to neuraxial morphine for the treatment of postoperative pain. Pain Research and Treatment 2012; 2012:612145

[85] Rathmell JP, Lair TR, Nauman B. The role of intrathecal drugs in the treatment of acute pain. Anesthesia \& Analgesia 2005; 101(5 Suppl), S30-S43

[86] Meylan N, Elia, Lysakowski, Tramèr MR. Benefit and risk of intrathecal morphine without local anaesthetic in patients undergoing major surgery: meta-analysis of randomized trials. British Journal of Anaesthesia 2009; 102(2) 156-67

[87] Elia N, Lysakowski C, Tramèr MR. Does multimodal analgesia with acetaminophen, no steroidal anti-inflammatory drugs, or selective cyclooxygenase-2 inhibitors and patient-controlled analgesia morphine offer advantages over morphine alone? Metaanalyses of randomized trials. Anesthesiology 2005; 103(6) 1296-1304

[88] Remy C, Marret E, Bonnet F. Effects of acetaminophen on morphine side effects and consumption after major surgery: meta-analysis of randomized controlled trials. British Journal of Anaesthesia 2005; 94(4) 505-513

[89] Engelman E, Marsala C. Efficacy of adding clonidine to intrathecal morphine in acute postoperative pain: a meta-analysis. British Journal of Anaesthesia 2013; 110(1) 21-7

[90] Liu SS, Richman JM, Thyrby RC, Wu CL. Efficacy of continuous wound catheters delivering local anesthetic for postoperative analgesia: a quantitative and qualitative systematic review of randomized controlled trials. Journal of the American College of Surgeons 2006; 203(6) 914-932 
[91] Gupta A, Favaios S, Perniola A, Magnuson A, Berggren L. A meta-analysis of the efficacy of wound catheters for postoperative pain management. Acta Anaesthesiologica Scandinavica 2011; 55(7) 785-796

[92] Rawal N, Borgeat A, Scott N. Wound catheters for postoperative pain: overture or finale? Acta Anaesthesiologica Scandinavica 2012; 56(3) 395-396

[93] Bertoglio S, Fabiani F, Negri PD, Corcione A, Merlo DF, Cafiero F, et al. The Postoperative Analgesic Efficacy of Preperitoneal Continuous Wound Infusion Compared to Epidural Continuous Infusion with Local Anesthetics after Colorectal Cancer Surgery: A Randomized Controlled Multicenter Study. Anesthesia \& Analgesia 2012; 115(6) 1442-50

[94] Procedure-Specific Postoperative Pain Management (PROSPECT). Available at: www.postoppain.org. Accessed July 18, 2013

[95] Practice guidelines for acute pain management in the perioperative setting. An updated report by the American Society of Anesthesiologists Task Force on Acute Pain Management. Anesthesiology 2012; 116(2) 248-273

[96] Curatolo M, Sveticic G. Drug combinations in pain treatment: a review of the published evidence and a method for finding the optimal combination. Best Practice \& Research Clinical Anaesthesiology 2002; 16(4) 507-519

[97] Santeularia MT, Catalá E, Genové M, Revuelta M, Moral MV. New trends in the treatment of postoperative pain in general and gastrointestinal surgery. Cirugía Española 2009; 86(2) 63-71

[98] White PF, Ofelia L. The role of multimodal analgesia in pain management after ambulatory surgery. Current Opinion in Anaesthesiology 2010; 23(6) 697-703.

[99] Joshi GP. Multimodal analgesia techniques and postoperative rehabilitation. Anesthesiology Clinics of North America 2005; 23(1) 185-202

[100] Varadhan KK, Neal KR, Dejong CHC, Fearon CH, Ljungqvist O, Lobo DN. The enhanced recovery after surgery (ERAS) pathway for patients undergoing major elective open colorectal surgery: a meta-analysis of randomized trials. Clinical Nutrition 2010; 29(4) 434-440

[101] Spanjersberg WR, Reurings J, Keus F, van Laarhoven CJ. Fast track surgery versus conventional recovery strategies for colorectal surgery. Cochrane Database of Systematic Reviews 2011; 2: CD007635

[102] Liu SS, Wu CL. Effect of postoperative analgesia on major postoperative complications: a systematic update of the evidence. Anesthesia \& Analgesia 2007; 104(3) 689-702

[103] Rawal N. Epidural technique for postoperative pain: gold standard no more? Regional Anesthesia and Pain Medicine 2012; 37(3) 310-7. 

